\title{
Modeling and Analysis of Chaos and Bifurcations for the Attitude System of a Quadrotor Unmanned Aerial Vehicle
}

\author{
Haiyun Bi, ${ }^{1,2}$ Guoyuan Qi ${ }^{3},^{3}$ and Jianbing $\mathrm{Hu}^{1}$ \\ ${ }^{1}$ School of Mechanical Engineering, Tianjin Polytechnic University, Tianjin 300384, China \\ ${ }^{2}$ The Key Laboratory of Advanced Perception and Intelligent Control of High-end Equipment, \\ Ministry of Education, and School of Mathematics and Physics, Anhui Polytechnic University, Wuhu 241000, China \\ ${ }^{3}$ Tianjin Key Laboratory of Advanced Technology of Electrical Engineering and Energy, Tianjin Polytechnic University, \\ Tianjin 300384, China
}

Correspondence should be addressed to Guoyuan Qi; guoyuanqisa@qq.com

Received 15 June 2019; Accepted 23 August 2019; Published 13 October 2019

Academic Editor: Carlos F. Aguilar-Ibáñez

Copyright (c) 2019 Haiyun Bi et al. This is an open access article distributed under the Creative Commons Attribution License, which permits unrestricted use, distribution, and reproduction in any medium, provided the original work is properly cited.

A model of the attitude system for a quadrotor unmanned aerial vehicle (QUAV), assumed to be a rigid body, is developed. For specific parameter configurations, a chaotic region with a saddle and two stable node-focus equilibrium points is identified. The chaotic model provides an important reference for dynamic analysis and a challengeable task of controller design once the flight enters the chaotic region of parameters. The pitchfork bifurcation of the equilibrium points is provided. Rich dynamics of the system are revealed by two bifurcation regions, which demonstrates the diversity of the flight behaviors as the parameters vary. One bifurcation analysis is with respect to the speed of the front propeller and the speed difference of the front and left propellers, and another one is with respect to the speed of the front propeller and moment of inertia. The dynamic characteristics of the QUAV are further verified by the Casimir power bifurcations. The trajectories of three settings with different structural parameters are analyzed in detail. The stability of the QUAV is found to be enhanced for certain optimized values of the structural parameters. Finally, using the Casimir power and Lagrange multiplier method, a supremum bound of the chaotic attractor is presented.

\section{Introduction}

The quadrotor unmanned aerial vehicle (QUAV) is a multirotor craft capable of autonomous control of its flight. Because of its small scale, low cost, flexible maneuverability, and high payload capability, it has specific advantages. Very recently, QUAVs have aroused great interest among many researchers around the world, including those in industries, academia, and governments [1]. This popularity is due to their wide range of applications such as military fighting, search and rescue missions, law enforcement, aerial cinematography, power-plant inspection, and agricultural and forestry spraying and supervision [2].

In practical applications, the spatial position of a QUAV is typically controlled by an operator via a remote-control system that uses visual feedback through an onboard camera and GPS, whereas the attitude (referring to the angular attitude in the whole paper) of a QUAV is automatically operated via an onboard controller. The attitude controller allows the QUAV to maintain a desired orientation, thus preventing the vehicle from flipping and bumping when the pilot performs the required maneuvers [2,3]. Currently, research on QUAVs is mainly dedicated to the design and improvement of the control algorithms, a large part of which is committed to the control of the QUAV attitude (see, for example, [3-11]).

Many scholars are dedicated to optimizing the control algorithms and exploring the strong external forces to stabilize the closed-loop system of the QUAV regardless of its characteristics. To the best of our knowledge, the literature concerning the dynamic analysis of the attitude of the QUAV is scant. Understanding the dynamic characteristics of a QUAV attitude system before designing and control is necessary. In addition, it is significant to study how the energy exchanges on the dynamics and the parameter configurations influence different dynamical behaviors (for 
instance, sink, periodic orbit, quasiperiodic orbit, and chaos). Furthermore, the dynamic analysis directs the design of the controller to achieve a desired performance. If a strong controller was designed without considering the characteristics of an open-loop QUAV, the control would expend energy in suppressing the oscillations, and such a controller would damage the actuator through the crude use of force. Building a dynamic model of a QUAV and studying its dynamic analysis are therefore significant in both dynamic research on chaos and practical applications.

Current modeling is mainly divided into mechanism modeling and system identification modeling. The former requires ample knowledge of the QUAV flight dynamics, which include flight mechanics, rigid dynamics, aerodynamics, and flipping angle dynamics. The latter needs data acquisition during flight testing to extract a simulation model. The method of acquisition is relatively simple and does not require a prior knowledge of the system dynamics. At present, the models established have generally six degrees of freedom. Some researchers used mechanical modeling $[8,9,12-17]$, and others combined methods using mechanical modeling first and then using system identification techniques to obtain the parameters $[3,18]$. Initially, dynamic models-linearized or otherwise-were developed, ignoring one or more effects of internal forces, air drag, and gyroscopic effects. Recently, literature studies have thrown up models that are considering more of the influencing factors including internal force, air drag, gyroscopic effects, and active inputs [14-16].

Although these models demonstrate very good results with their control algorithms, the literature contains little concerning the dynamic analysis of the QUAV attitude system. There has also been scant research on the chaotic behavior of the QUAV attitude system. Why there is lack of research on QUAV chaos? One of the main variables of the QUAV attitude system is angular velocity. However, observations are made with angles rather than angular velocity. Angles are associated with integrals of angular velocities, and therefore, the former are much smoother than the latter. Angles are evaluated and measured but do not necessarily undergo chaotic behavior if the angular velocities behave chaotically. According to the studies reported in [19-21], angular velocities usually display chaotic modes for a forceddissipative rigid body, the description of which is given by the Kolmogorov model.

The literature studies concerning the dynamic analysis of the attitude of the QUAV are scant; therefore, we can refer to the research of many other physical forced-dissipative rigid or generalized rigid systems exhibiting chaotic motion under some parameter configurations or disturbances, for instance, the chaotic system of a brushless DC motor (BLDCM) [22] and the chaos and bifurcation of a permanent magnet synchronous motor (PMSM) [23]. Although both the motors are electromechanical systems, they can be regarded as generalized rigid bodies for the study of their dynamics (see in particular $[24,25])$. Moreover, many numerical chaotic systems were converted to resemble rigid-body systems by transforming the system to the Kolmogorov formalism. Details of the nature of the mechanism or energy were thus revealed [26-30]. The satellite is also a rigid body that within certain regions in its parameter space displays chaotic behavior. Kuang et al. [19] gave a chaotic analysis of the attitude of the satellite under small disturbances of its moments. Faramin and Ataei [20] analyzed the chaos of the satellite attitude via the Lyapunov exponents (LEs), designed a nonlinear robust control to suppress chaos, and confirmed its suppression using Melnikov's analysis. Generally, the Melnikov method affords the necessary conditions for the existence of chaotic motion [31-34]. In dynamic research, a typical rigid body is the gyrostat. Doroshin [21] presented a model of a gyroscopic system and analyzed its strange attractors. Qi [35] modeled a class of generalized 4D Euler's rigid systems and then presented the mechanical mechanism of a derived Hamiltonian conservative chaotic system that exhibits strong ergodicity. As the QUAV is a very typical rigid-body system, basic dynamic analysis of the attitude system of a QUAV is meaningful for controller design.

In this paper, a dimensionalized attitude system of a rigid-body QUAV describing real yaw maneuvers is developed. By adjusting the values of some structural parameters and the rotor speeds through thrusts, the angular velocities of the QUAV attitude system demonstrate chaotic behavior. The significances of this modeling and findings on the QUAV are as follows: (1) a new physical chaotic model is provided for future research of dynamic, bifurcation analysis; (2) the chaos finding and investigation provide the important warning that when configurations of bobweight, moment of inertia, and speeds of front and rear propellers, etc. are improperly designed, the flight has great potentiality entering into the region of chaos or oscillation which brings about a great difficulty to control; and (3) it is a much more challengeable task for controller design once the QUAV takes on the chaotic behavior than the ordinary flight.

It is found that the chaotic system has a saddle and two stable node-focus equilibrium points for some parameter configurations and rotor speeds. There are few chaotic systems with equilibrium points of these stability types, especially for physical systems with actual backgrounds. These types of equilibria have the possibility to produce hidden attractors. This again increases the potentiality of danger during the flight when the QUAV is disturbed by wind, thereby entering the hidden region. The pitchfork bifurcation of equilibrium points is provided in this paper. Rich dynamics of the system are uncovered via two bifurcation regions, which demonstrates the diversity of the flight behavior when the parameters vary. One bifurcation region displays dynamics with respect to the speed of the front propeller and the speed difference of the front and left propellers. Another bifurcation region shows dynamics of the speed of the front propeller and moment of inertia with respect to the $x$-axis. Regions in the parameter space that are susceptible to chaos in the hovering state are analyzed in detail. The dynamic characteristics of the QUAV are further verified by the Casimir power bifurcations. The energy exchange, energy change rate, and reasons for the formation of the dynamic characteristics of the system are revealed via Casimir energy and Casimir power. The trajectories of three settings with different structural parameters are analyzed in 
detail. The stability of the QUAV is found to be enhanced for certain optimized values of the structural parameters. The results of this dynamic analysis are helpful in the design of the shape and bobweight of a QUAV and in setting the direction of the driving force and its magnitude. This research also helps to suppress any large fluctuations in speed and chaotic motion in the case of yaw movement because of incorrect configurations of the moments of inertia and other system parameters. At last, with the Lagrange multiplier technique and Casimir power, an analytical supremum bound of the chaotic attractor is given. The boundary of the system can help to comprehend the global dynamics of the system, such as indicating that the system's total energy and amplitude of the variable cannot grow unboundedly.

The rest of this paper is organized as follows: The model of the QUAV attitude system is provided in Section 2. The pitchfork bifurcation is plotted in Section 3. The detailed dynamic analysis is given in Section 4 . The supremum bound of the chaotic attractor is proposed in Section 5. Section 6 presents our conclusions.

\section{Quadrotor Dynamic Model}

A full description of the motion of the quadrotor requires two reference frames (Figure 1), specifically the earth's inertial frame (E-frame) and the body-fixed frame (B-frame).

To set up the dynamic model of the QUAV, the primary assumptions are as follows [36].

Assumption 1. The QUAV is considered a strictly rigid body.

Assumption 2. The configuration of the quadrotor is completely symmetrical with respect to the $x-z$ and $x-y$ planes, and the inertia matrix is time invariant.

Assumption 3. Thrusts are proportional to the square of the propeller speed.

The mathematical model of the QUAV has six degrees of freedom and can be divided into three parts: attitude dynamics, kinematic model, and position dynamics. The force relationship among the three dynamical subsystems is illustrated in Figure 2. The dynamic equations for the QUAV are $[15,16]$

$$
\left\{\begin{array}{l}
m \ddot{\xi}=F_{\mathrm{f}}-F_{\mathrm{d}}-F_{\mathrm{g}}, \\
I \dot{\boldsymbol{\omega}}=-S(I \boldsymbol{\omega}) \boldsymbol{\omega}+\Gamma_{\alpha}-\Gamma_{\mathrm{d}}+\Gamma_{\mathrm{f}} .
\end{array}\right.
$$

The first equation of system (1)

$$
m \ddot{\xi}=F_{\mathrm{f}}-F_{\mathrm{d}}-F_{\mathrm{g}},
$$

is called the QUAV position system, where $m$ denotes the total mass of the QUAV and $\xi=\left[\begin{array}{lll}x & y & z\end{array}\right]^{\mathrm{T}}$ the position of the center of mass of the QUAV in the E-frame. The term $F_{\mathrm{f}}$ represents the resultant force generated by the four propellers of the QUAV and is expressed as

$$
F_{\mathrm{f}}=\left[\begin{array}{c}
\cos \phi \sin \theta \cos \psi+\sin \phi \sin \psi \\
\cos \phi \sin \theta \sin \psi-\sin \phi \cos \psi \\
\cos \phi \cos \theta
\end{array}\right] F_{\mathrm{T}},
$$

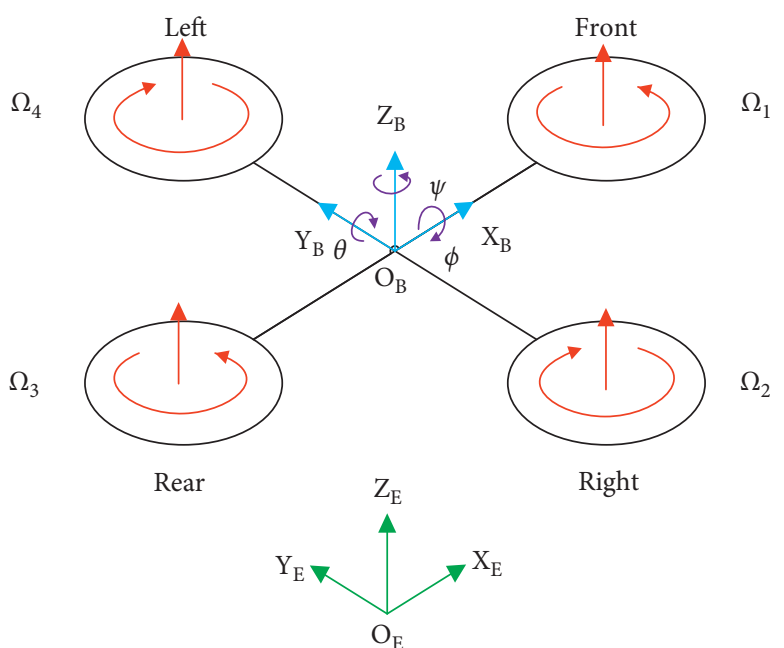

FIgURE 1: Schematic view of the QUAV system.

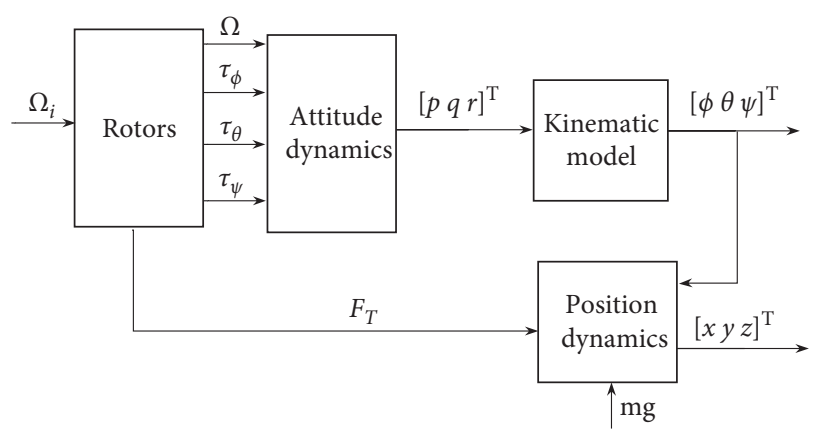

FIGURE 2: Force relationship diagram of dynamics of subsystems of the QUAV.

where $F_{\mathrm{T}}=\sum_{i=1}^{4} b \Omega_{i}^{2}$, with coefficient $b$ relating to lift and $\Omega_{i}(j=1,2,3,4)$ the rotational speed of the $j$-th propeller of the QUAV (Figure 1). Angles $\phi, \theta$, and $\psi$ are related to roll, pitch, and yaw, respectively. $\Theta=\left[\begin{array}{lll}\phi & \theta & \psi\end{array}\right]^{\mathrm{T}}$ is the vector of angular attitude with respect to the E-frame. The term $F_{\mathrm{d}}$ represents the resultant force generated along the $x$-, $y$-, and $z$-axes:

$$
F_{\mathrm{d}}=\left[\begin{array}{ccc}
k_{1} & 0 & 0 \\
0 & k_{2} & 0 \\
0 & 0 & k_{3}
\end{array}\right]\left[\begin{array}{c}
\dot{x} \\
\dot{y} \\
\dot{z}
\end{array}\right],
$$

where $k_{1}, k_{2}$, and $k_{3}$ are translational drag coefficients. The gravitational force $F_{\mathrm{g}}$ is written as

$$
F_{\mathrm{g}}=\left[\begin{array}{lll}
0 & 0 & \mathrm{mg}
\end{array}\right]^{\mathrm{T}} \text {. }
$$

Then, the QUAV position dynamic system is specified as

$$
\left\{\begin{array}{l}
m \ddot{x}=F_{\mathrm{T}}(\cos \phi \sin \theta \cos \psi+\sin \phi \sin \psi)-k_{1} \dot{x}, \\
m \ddot{y}=F_{\mathrm{T}}(\cos \phi \sin \theta \sin \psi-\sin \phi \cos \psi)-k_{2} \dot{y}, \\
m \ddot{z}=F_{\mathrm{T}} \cos \phi \cos \theta-k_{3} \dot{z}-\mathrm{mg} .
\end{array}\right.
$$

The second equation of system (1)

$$
I \dot{\boldsymbol{\omega}}=-S(I \boldsymbol{\omega}) \boldsymbol{\omega}+\Gamma_{\alpha}-\Gamma_{\mathrm{d}}+\Gamma_{\mathrm{f}},
$$


is called the QUAV attitude system. Here, $I \in R^{3 \times 3}$ is a symmetrical positive-definite constant matrix associated with the inertia of the QUAV with respect to the B-frame and is defined as

$$
I=\left[\begin{array}{ccc}
I_{x} & 0 & 0 \\
0 & I_{y} & 0 \\
0 & 0 & I_{z}
\end{array}\right]
$$

where $I_{x}, I_{y}$, and $I_{z}$ are the three moments of inertia with respect to the $x$-, $y$-, and $z$-axes. The vector $\boldsymbol{\omega}=\left[\begin{array}{lll}p & q & r\end{array}\right]^{\mathrm{T}}$ is the vector of attitude angular velocity with respect to the B-frame, and $S(I \boldsymbol{\omega})$ is an antisymmetric matrix specified as

$$
S(I \boldsymbol{\omega})=-S^{T}(I \boldsymbol{\omega})=\left[\begin{array}{ccc}
0 & I_{z} r & -I_{y} q \\
-I_{z} r & 0 & I_{x} p \\
I_{y} q & -I_{x} p & 0
\end{array}\right] .
$$

The term $\Gamma_{\alpha}$ denotes the resultant moment generated by the gyroscopic effect:

$$
\Gamma_{\alpha}=I_{r}\left[\begin{array}{c}
-q \\
p \\
0
\end{array}\right] \Omega
$$

where $I_{r}$ is the total rotational moment of inertia around the propeller of the QUAV and $\Omega$ is the speed of the rotorcraft which is defined as $\Omega=\Omega_{2}+\Omega_{4}-\Omega_{1}-\Omega_{3}$. The term $\Gamma_{\mathrm{d}}$ denotes the rotational translational drag torque:

$$
\Gamma_{\mathrm{d}}=\left[\begin{array}{ccc}
k_{4} & 0 & 0 \\
0 & k_{5} & 0 \\
0 & 0 & k_{6}
\end{array}\right]\left[\begin{array}{l}
p \\
q \\
r
\end{array}\right],
$$

where $k_{i}(i=4,5,6)$ are the positive drag coefficients for the rotor dynamics. The term $\Gamma_{\mathrm{f}}$ is the torque associated with the thrust generated by the active input and has the following form:

$$
\Gamma_{\mathrm{f}}=\left[\begin{array}{c}
\tau_{\phi} \\
\tau_{\theta} \\
\tau_{\psi}
\end{array}\right]=\left[\begin{array}{c}
b l\left(\Omega_{4}^{2}-\Omega_{2}^{2}\right) \\
b l\left(\Omega_{3}^{2}-\Omega_{1}^{2}\right) \\
d\left(\Omega_{4}^{2}+\Omega_{2}^{2}-\Omega_{3}^{2}-\Omega_{1}^{2}\right)
\end{array}\right],
$$

where $l$ denotes the distance from the center of the QUAV to the center of the rotation axis of the propeller and $d$ the drag coefficient.

Remark 1. The vector field of equation (7), the attitude system of the QUAV, is a force field constituted from four torques: inertial torque, $-S(I \boldsymbol{\omega}) \boldsymbol{\omega}$; internal torque (often called the gyro effect), $\Gamma_{\alpha}$; dissipative torque, $-\Gamma_{d}$; and external torque, $\Gamma_{\mathrm{f}}$. This decomposition of the total torque conforms with the Kolmogorov system; see references [24, 25, 37].

The QUAV attitude system is specified as

$$
\left\{\begin{array}{l}
I_{x} \dot{p}=\left(I_{y}-I_{z}\right) q r-I_{r} \Omega q-k_{4} p+\tau_{\phi}, \\
I_{y} \dot{q}=\left(I_{z}-I_{x}\right) p r+I_{r} \Omega p-k_{5} q+\tau_{\theta}, \\
I_{z} \dot{r}=\left(I_{x}-I_{y}\right) p q-k_{6} r+\tau_{\psi} .
\end{array}\right.
$$

This system is the core subsystem of the QUAV system (Figure 2).

To implement yaw maneuvers, the speeds of the front and rear propellers should be equal, i.e., $\Omega_{1}=\Omega_{3}$ (Figure 1), as must the speeds of the left and right propellers, i.e., $\Omega_{2}=\Omega_{4}$. If the speeds of the front and left propellers differ, $c=\Omega_{2}-\Omega_{1} \neq 0$, a countertorque is produced. Furthermore, keeping the overall thrust equal to the force of gravity on the quadrotor, a yaw maneuver occurs when hovering. For the QUAV, the yaw maneuver meets the conditions

$$
\begin{aligned}
& \tau_{\phi}=0, \\
& \tau_{\theta}=0, \\
& \Omega=\Omega_{2}+\Omega_{4}-\Omega_{1}-\Omega_{3}=2 c \neq 0 .
\end{aligned}
$$

Substituting these conditions into equation (13), the QUAV attitude system under yaw maneuver is simplified to

$$
\left\{\begin{array}{l}
I_{x} \dot{p}=\left(I_{y}-I_{z}\right) q r-2 c I_{\mathrm{r}} q-k_{4} p, \\
I_{y} \dot{q}=\left(I_{z}-I_{x}\right) p r+2 c I_{\mathrm{r}} p-k_{5} q, \\
I_{z} \dot{r}=\left(I_{x}-I_{y}\right) p q-k_{6} r+2 d\left(c^{2}+2 c \Omega_{1}\right) .
\end{array}\right.
$$

Next, we investigate the dynamic characteristics and mechanical analysis of system (15). The relationship between the vector of attitude angular velocity $\dot{\Theta}=\left[\begin{array}{lll}\dot{\phi} & \dot{\theta} & \dot{\psi}\end{array}\right]^{\mathrm{T}}$ with respect to the $\mathrm{E}$-frame and the vector of attitude angular velocity $\boldsymbol{\omega}=\left[\begin{array}{lll}p & q & r\end{array}\right]^{\mathrm{T}}$ with respect to the $\mathrm{B}$-frame is expressed as

$$
\left[\begin{array}{c}
\dot{\phi} \\
\dot{\theta} \\
\dot{\psi}
\end{array}\right]=\left[\begin{array}{ccc}
1 & \sin \phi \tan \theta & \cos \phi \tan \theta \\
0 & \cos \phi & -\sin \phi \\
0 & \frac{\sin \phi}{\cos \theta} & \frac{\cos \phi}{\cos \theta}
\end{array}\right]\left[\begin{array}{l}
p \\
q \\
r
\end{array}\right] .
$$

The detailed relationship between the angular velocity vector with respect to the E-frame and the angular velocity vector with respect to the B-frame was explained in reference [38].

\section{Equilibrium of the QUAV Attitude System during Yaw Maneuvers}

Let $\dot{\boldsymbol{\omega}}=\left[\begin{array}{lll}\dot{p} & \dot{q} & \dot{r}\end{array}\right]^{\mathrm{T}}=0$, the equilibrium points of system (15) are then completely stated as

$$
\begin{aligned}
& E_{1}=\left[\begin{array}{lll}
0 & 0 & a_{15}
\end{array}\right]^{\mathrm{T}},
\end{aligned}
$$

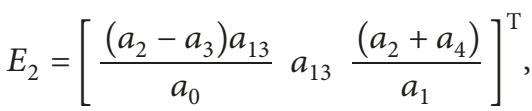

$$
\begin{aligned}
& E_{3}=\left[-\frac{\left(a_{2}-a_{3}\right) a_{13}}{a_{0}}-a_{13} \frac{\left(a_{2}+a_{4}\right)}{a_{1}}\right]^{\mathrm{T}} \text {, } \\
& E_{4}=\left[-\frac{\left(a_{2}+a_{3}\right) a_{14}}{a_{0}} a_{14}-\frac{a_{2}-a_{4}}{a_{1}}\right]^{\mathrm{T}} \text {, } \\
& E_{5}=\left[\frac{\left(a_{2}+a_{3}\right) a_{14}}{a_{0}}-a_{14}-\frac{\left(a_{2}-a_{4}\right)}{a_{1}}\right]^{\mathrm{T}} \text {, }
\end{aligned}
$$


where

$$
\begin{aligned}
& a_{0}=k_{4}\left(I_{x}-I_{z}\right), \\
& a_{1}=\left(I_{x}-I_{z}\right)\left(I_{y}-I_{z}\right), \\
& a_{2}=\sqrt{\left(\left(I_{x}-I_{y}\right) I_{\mathrm{r}} c\right)^{2}-a_{1} k_{4} k_{5},} \\
& a_{3}=\left(I_{x}-I_{y}\right) I_{r} c, \\
& a_{4}=\left(I_{x}+I_{y}-2 I_{z}\right) I_{r} c, \\
& a_{5}=\left(I_{x}-I_{y}\right)\left(I_{\mathrm{r}} c\right)^{2} k_{6}, \\
& a_{15}=2\left(I_{x}-I_{y}\right)\left(I_{y}-I_{z}\right) \mathrm{d} I_{r} c^{3}, \\
& a_{13}=\sqrt{\frac{\left(-a_{5}+a_{6}+a_{7}+a_{8}-a_{9}-a_{10}+a_{11}\right)}{a_{12}}} \\
& a_{7}=4\left(I_{x}-I_{y}\right)\left(I_{y}-I_{z}\right) \mathrm{d} \Omega_{1} I_{r} c^{2}, \\
& a_{10}=4\left(I_{y}-I_{z}\right) a_{2} \mathrm{~d} \Omega_{1} c, \\
& a_{9}=2 k_{4} k_{5} k_{6}, \\
& a_{2} k_{6} I_{r} c, \\
& a_{12}\left(I_{y}-I_{z}\right) a_{2} \mathrm{~d} c^{2},
\end{aligned}
$$

From equation (18), the signs of $a_{2}^{2}, a_{13}^{2}$, and $a_{14}^{2}$ are key in determining the existence of equilibrium points. Looking at Table 1, we can see there exists a pitchfork bifurcation at which

\begin{tabular}{|c|c|c|c|c|}
\hline Parameter & $a_{2}^{2}$ & $a_{13}^{2}$ & $a_{14}^{2}$ & Equilibrium \\
\hline \multirow{5}{*}{ Sign condition } & - & Any sign & Any sign & $E_{1}$ \\
\hline & + & - & - & $E_{1}$ \\
\hline & + & + & - & $E_{1}, E_{2}, E_{3}$ \\
\hline & + & - & + & $E_{1}, E_{4}, E_{5}$ \\
\hline & + & + & + & $E_{1}, E_{2}, E_{3}, E_{4}, E_{5}$ \\
\hline
\end{tabular}
these equilibrium points might undergo transitions from one equilibrium point to three equilibrium points and from three to five.

The values of the parameters used below are taken from references [3, 8], which were acquired from a modified version of the QUAV Draganflyer III. All of its parameter values are listed in Table 2, which we adopt along with setting $c=5 \mathrm{rad} / \mathrm{s}$ to investigate the bifurcation characteristics of yaw maneuvers.

In accordance with the transition condition of the equilibrium points (Table 1), the speed of the front propeller $\Omega_{1}$ is selected as an independent variable, adopting the values of all other parameters in Table 2 . There is a transition
TABLE 1: Numbers of equilibrium points for various parameter signs.

TABle 2: Parameter values of the quadrotor $[3,8]$.

\begin{tabular}{lc}
\hline Parameter & Value \\
\hline$g\left(\mathrm{~m} / \mathrm{s}^{2}\right)$ & 9.81 \\
$m(\mathrm{~kg})$ & 0.468 \\
$l(\mathrm{~m})$ & 0.225 \\
$b(\mathrm{~N} \cdot \mathrm{m} / \mathrm{rad} / \mathrm{s})$ & $2.9 e-5$ \\
$d(\mathrm{~m})$ & $1.1 e-6$ \\
$I_{\mathrm{r}}\left(\mathrm{kg} \cdot \mathrm{m}^{2}\right)$ & $3.4 e-5$ \\
$I_{x}\left(\mathrm{~kg} \cdot \mathrm{m}^{2}\right)$ & $4.9 e-3$ \\
$I_{y}\left(\mathrm{~kg} \cdot \mathrm{m}^{2}\right)$ & $9.8 e-3$ \\
$I_{z}\left(\mathrm{~kg} \cdot \mathrm{m}^{2}\right)$ & $8.8 e-3$ \\
$k_{1}(\mathrm{~N} / \mathrm{m} / \mathrm{s})$ & $2.45 e-4$ \\
$k_{2}(\mathrm{~N} / \mathrm{m} / \mathrm{s})$ & $8.82 e-4$ \\
$k_{3}(\mathrm{~N} / \mathrm{m} / \mathrm{s})$ & $4.40 e-4$ \\
$k_{4}(\mathrm{~N} / \mathrm{rad} / \mathrm{s})$ & $2.45 e-4$ \\
$k_{5}(\mathrm{~N} / \mathrm{rad} / \mathrm{s})$ & $8.82 e-4$ \\
$k_{6}(\mathrm{~N} / \mathrm{rad} / \mathrm{s})$ & $4.40 e-4$ \\
\hline
\end{tabular}

that the equilibrium points undergo: a transition from one to three equilibrium points and a transition in the speed of the front propeller at $\Omega_{1}=6.3852 \mathrm{rad} / \mathrm{s}$. A calculation shows that it is impossible for $a_{2}^{2}, a_{13}^{2}$, and $a_{14}^{2}$ to be positive at the same time; that is, $a_{2}, a_{13}$, and $a_{14}$ cannot be real numbers simultaneously, and therefore, the transition from three to five does not occur with these parameter settings. Hence, there are mainly three equilibrium points for the yaw maneuver.

The pitchfork bifurcation diagram of the yaw maneuver is shown in Figure 3. The changes of stability of equilibrium points $E_{1}, E_{2}$, and $E_{3}$ with respect to parameter $\Omega_{1}$ are displayed. When $\Omega_{1} \in(0,6.3852)(\mathrm{rad} / \mathrm{s})$, there is only one stable equilibrium point $E_{1}$. When $\Omega_{1} \in(6.3852,200)(\mathrm{rad} / \mathrm{s}), E_{1}$ changes its stability type to be saddle and $E_{2}$ and $E_{3}$ become stable nodefoci. The QUAV attitude system (15) encounters pitchfork bifurcation near $\Omega_{1}=6.3852 \mathrm{rad} / \mathrm{s}$. When a pitchfork bifurcation takes place, the QUAV undergoes some complicated dynamics. The system orbit may enter into either one of two undesired stable equilibrium points or run periodically, multiperiodically, or even chaotically. This is the hidden difficulty with the system.

The Casimir function, $C(\mathbf{x})$, also referred to as the energy-momentum $[24,39]$, is a significant physical quantity in chaotic system analysis $[24,25,37]$. In the QUAV attitude system, let $\mathbf{x}=\sqrt{I} \boldsymbol{\omega}=\left[\begin{array}{lll}\sqrt{I_{x}} p & \sqrt{I_{y}} q & \sqrt{I_{z}} r\end{array}\right]^{\mathrm{T}}$ and $\boldsymbol{\omega}=$ $\left[\begin{array}{lll}p & q & r\end{array}\right]^{\mathrm{T}}$ be angular velocity. $C(\mathbf{x})=C(\sqrt{I} \boldsymbol{\omega})$ represents the rotational kinetic energy, i.e.,

$$
\begin{aligned}
C(\mathbf{x}) & =\frac{1}{2}\langle\mathbf{x}, \mathbf{x}\rangle=\frac{1}{2}\left(x_{1}^{2}+x_{2}^{2}+x_{3}^{2}\right) \\
& =C(\sqrt{I} \boldsymbol{\omega})=\frac{1}{2}\left(I_{x} p^{2}+I_{y} q^{2}+I_{z} r^{2}\right) .
\end{aligned}
$$




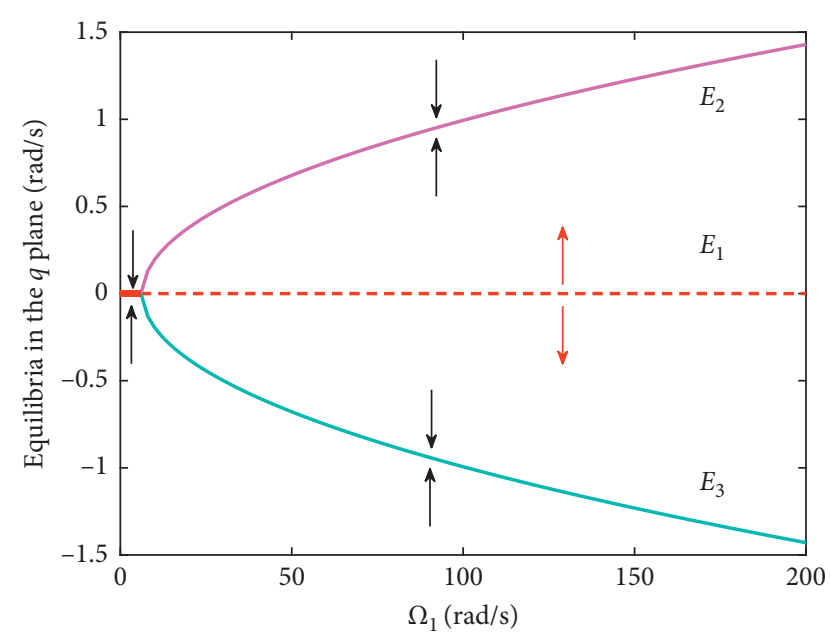

FIGURE 3: Pitchfork bifurcation of the equilibrium points.

The Casimir power, the rate of change of the Casimir energy, is

$$
\begin{aligned}
\dot{C}(\mathbf{x})= & \frac{\partial C}{\partial \mathbf{x}} \dot{\mathbf{x}}=x_{1} \dot{x}_{1}+x_{2} \dot{x}_{2}+x_{3} \dot{x}_{3} \\
= & \dot{C}(\sqrt{I} \boldsymbol{\omega})=I_{x} p \dot{p}+I_{y} q \dot{q}+I_{z} r \dot{r} \\
= & p\left[\left(I_{y}-I_{z}\right) q r-2 c I_{\mathrm{r}} q-k_{4} p\right]+q\left[\left(I_{z}-I_{x}\right) p r\right. \\
& \left.+2 c I_{r} p-k_{5} q\right]+r\left[\left(I_{x}-I_{y}\right) p q-k_{6} r+2 d\left(c^{2}+2 c \Omega_{1}\right)\right] \\
= & 2 d\left(c^{2}+2 c \Omega_{1}\right) r-\left(k_{4} p^{2}+k_{5} q^{2}+k_{6} r^{2}\right) .
\end{aligned}
$$

Note that the term $2 d\left(c^{2}+2 c \Omega_{1}\right) r$ is the supplied power provided by the propelling thrust and the term $\left(k_{4} p^{2}+\right.$ $\left.k_{5} q^{2}+k_{6} r^{2}\right)$ is the dissipated power lost due to air drag. Therefore, the Casimir power is the difference between the supplied power and the dissipated power.

Remark 2. Casimir energy, $C(\mathbf{x})$, is the rotational kinetic energy of the QUAV, with unit $\mathrm{kg} \cdot \mathrm{m}^{2} \cdot \mathrm{rad}^{2} / \mathrm{s}^{2}=\mathrm{N} \cdot \mathrm{m}=\mathrm{J}$. Casimir power, $\dot{C}(\mathbf{x})$, is the rotational power of the QUAV, with unit $W$.

Remark 3. From the Casimir energy and the Casimir power, we draw the following conclusions [24, 37]:

(1) If $\dot{C}(\mathbf{x})>0$ holds for all times, then the system's orbit diverges as a source.

(2) If $\dot{C}(\mathbf{x})$ is a nonzero constant, i.e., $\dot{C}(\mathbf{x}) \equiv 0$ with $C(\mathbf{x}) \neq 0$, then the orbit is periodic.

(3) If $\dot{C}(\mathbf{x})<0$ holds for all times, then the orbit converges to a sink.

(4) If $\dot{C}(\mathbf{x})$ oscillates periodically, then the orbit is periodic.

(5) If $\dot{C}(\mathbf{x})$ is bounded and irregularly vibrating around the zero line, then the orbit is chaotic.

(6) If $\dot{C}(\mathbf{x})$ converges to zero asymptotically, then the orbit converges to a sink.
Thus, the Casimir power provides criteria to determine whether a system produces chaotic motion.

In this section, stability analysis and a pitchfork bifurcation of the equilibrium points were presented. Casimir energy and Casimir power of the system were derived, which reflect the characteristics of the system trajectory. This will be demonstrated in the next section.

\section{Dynamic Analysis}

4.1. Basic Dynamic Analysis. In the following sections, we take the initial condition as $\boldsymbol{\omega}_{0}=\left[\begin{array}{lll}p_{0} & q_{0} & r_{0}\end{array}\right]^{\mathrm{T}}=$ $\left[\begin{array}{lll}0.001 & 0.001 & 0.001\end{array}\right]^{\mathrm{T}}(\mathrm{rad} / \mathrm{s})$. Detailed dynamic analyses of the system (15) via Lyapunov exponents, equilibrium point analysis, and bifurcation analyses are provided. Setting $c=$ $5 \mathrm{rad} / \mathrm{s}$ and $\Omega_{1}=30 \mathrm{rad} / \mathrm{s}$, and adopting the values of all other parameters in Table 2, the trajectory of system (15) falls towards a sink, the maximum LE $L_{1}=-0.00991$, and the Lyapunov dimension equals zero, matching observations (Figure 4(a)). When $c=5 \mathrm{rad} / \mathrm{s}$ and $\Omega_{1}=196.4 \mathrm{rad} / \mathrm{s}$, the orbit of system (15) is chaotic, the maximum $\operatorname{LE~} L_{1}=0.040392$, and the Lyapunov dimension equals 2.1753, confirming that the QUAV operates in a chaotic mode (Figure 4(b)). Although the chaotic mode is weak, because the body is very small, it still has a relatively large impact on the QUAV.

To comprehend the influence of the rotational speed parameter $\Omega_{1}$ and speed difference $c$ on the QUAV attitude system (15) during yaw maneuvers, we varied $\Omega_{1} \in$ $[50,500](\mathrm{rad} / \mathrm{s})$ and $c \in[3,7](\mathrm{rad} / \mathrm{s})$, leaving the remaining parameters as given in Table 2. The regions of various dynamical orbits are plotted in Figure 5. The blue area indicates trajectories of the system converging to a sink. This implies that, under this parameter configuration, the attitude of the QUAV is relatively stable and easy to control. The red region indicates trajectories that are chaotic, signifying that the angular velocity of the QUAV has irregular oscillations and is not conducive to control. The yellow region marks orbits that are periodic, but the QUAV is still unstable as periodic oscillations occur. Compared with the emergence of chaos, the periodic oscillations in the angular velocity are regular and relatively easy to control. We see that, with increasing rotor speed difference $c$, the trajectory of the QUAV is inclined towards chaos when the rotor speed $\Omega_{1}$ is relatively low.

Remark 4. The condition of the QUAV in hovering at the equilibrium point, $\phi=0 \mathrm{rad}, \theta=0 \mathrm{rad}$, and $\psi=0 \mathrm{rad}$, is

$$
F_{\mathrm{f}}=F_{\mathrm{T}}=\sum_{i=1}^{4} b \Omega_{i}^{2}=\mathrm{mg} .
$$

For the parameter values given in Table 2, we have the following relationship between the speed of the front rotor $\Omega_{1}$ and the rotor speed difference $c$ :

$$
2.9 e-5 \times\left[2 \Omega_{1}^{2}+2\left(\Omega_{1}+c\right)^{2}\right](\mathrm{N})=0.468 \times 9.81(\mathrm{~N}) .
$$

Setting the ranges $c \in[3,7](\mathrm{rad} / \mathrm{s})$ and $\Omega_{1} \in[50,500]$ $(\mathrm{rad} / \mathrm{s})$, when $c$ and $\Omega_{1}$ satisfy equation (22), the QUAV 


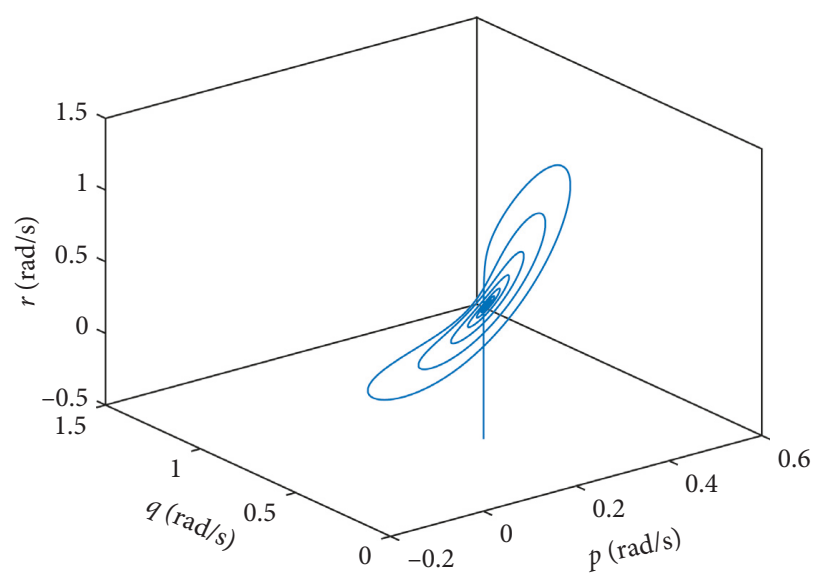

(a)

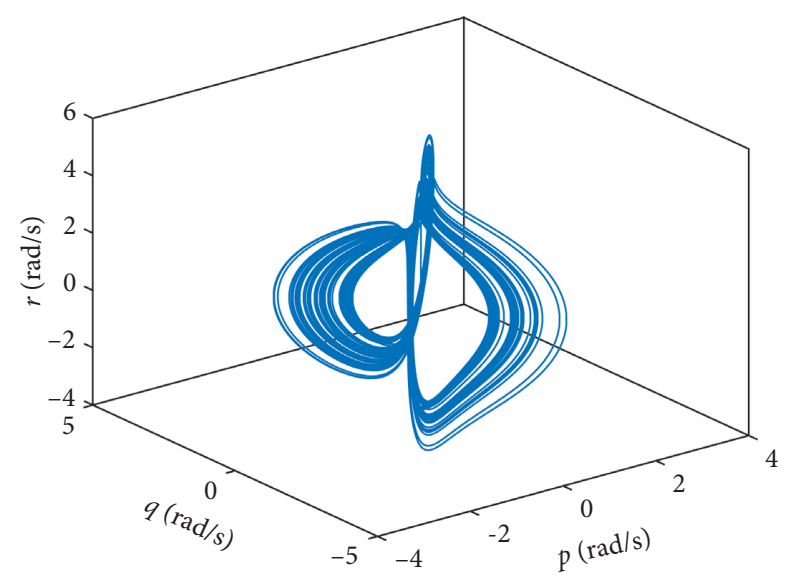

(b)

FIgURE 4: Trajectory of system (15): (a) sink; (b) chaos.

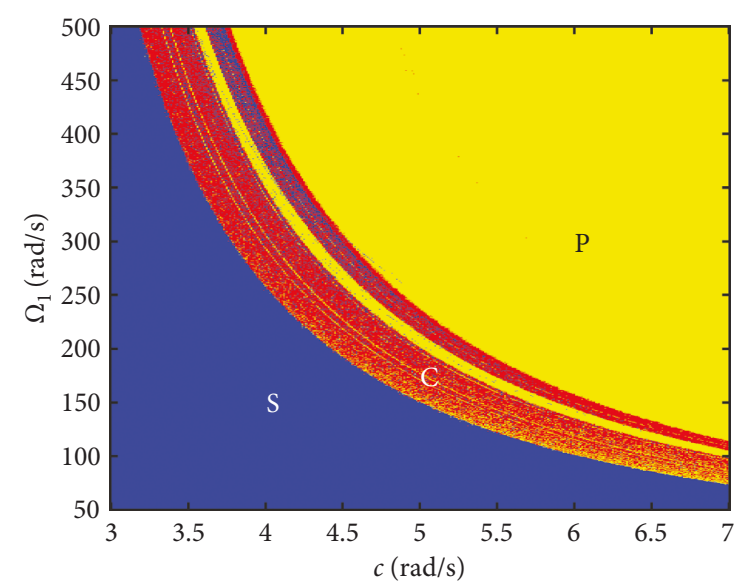

Figure 5: Three colored dynamical regions of system (15) at the initial value of $\boldsymbol{\omega}_{0}=\left[\begin{array}{lll}p_{0} & q_{0} & r_{0}\end{array}\right]^{\mathrm{T}}=\left[\begin{array}{lll}0.001 & 0.001 & 0.001\end{array}\right]^{\mathrm{T}}(\mathrm{rad} / \mathrm{s})$ with $c \in[3,7](\mathrm{rad} / \mathrm{s})$ and $\Omega_{1} \in[50,500](\mathrm{rad} / \mathrm{s})$.

hovers. The relationship between the two parameters is shown in Figure 6. Therefore, the yaw maneuver in the hovering state should be performed to avoid chaotic movements as much as possible. When $\Omega_{1} \in(197.4,500]$ $(\mathrm{rad} / \mathrm{s})$ and $c \in[3,7](\mathrm{rad} / \mathrm{s})$, the resultant force is in the range of $F_{\mathrm{f}} \in(4.591,29.175](\mathrm{N})$, which is larger than the force of gravity, $\mathrm{mg}=4.591 \mathrm{~N}$, and hence, the QUAV takes off. When $\Omega_{1} \in[195.4,197.4](\mathrm{rad} / \mathrm{s})$ and $c \in[4.5,5.2](\mathrm{rad} / \mathrm{s})$, the QUAV tends to behave chaotically in the hovering state (Figure 5).

The QUAV has rich dynamics as demonstrated macroscopically by the bifurcation diagram of the angular velocity component $p$ with respect to $\Omega_{1}$ with $c=5 \mathrm{rad} / \mathrm{s}$ (Figure 7). When $\Omega_{1} \in(0,147](\mathrm{rad} / \mathrm{s})$, the system's trajectory converges onto a sink. Note also that when $\Omega_{1}=40 \mathrm{rad} / \mathrm{s}$, the convergence point for the trajectory switches from sink $E_{2}$ to sink $E_{3}$. This can be seen more clearly from the phase portraits (Figures $8(a)$ and $8(\mathrm{c})$ ). Setting $\Omega_{1}=30 \mathrm{rad} / \mathrm{s}$, the trajectory converges to $E_{2}$ (Figure 8(b)); with the same initial point, but setting $\Omega_{1}=100 \mathrm{rad} / \mathrm{s}$, the trajectory approaches $E_{3}$ (Figure 8(c)).

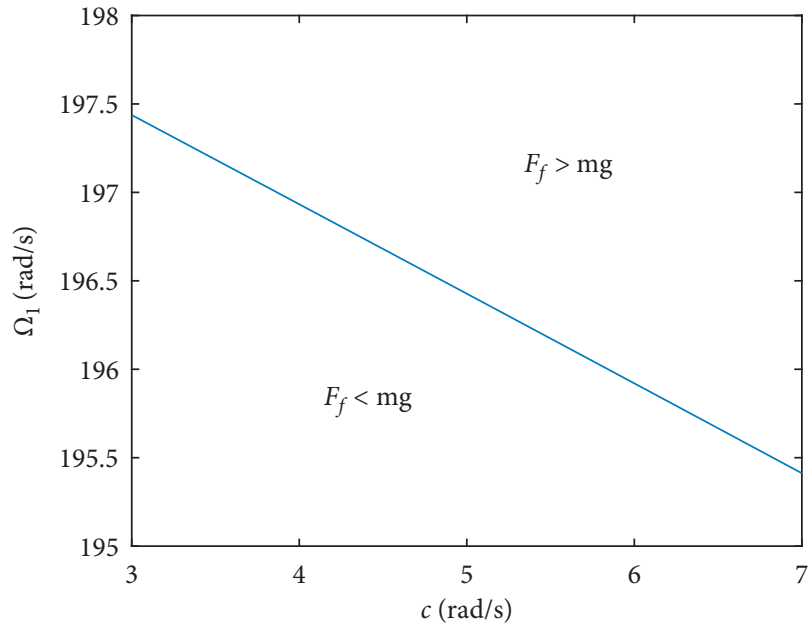

FIGURE 6: Relationship between $c$ and $\Omega_{1}$ in the hovering state of a QUAV.

The angular velocity of the system demonstrates chaotic behavior in the parameter range $\Omega_{1} \in(147,203) \cup$ $(216,244)(\mathrm{rad} / \mathrm{s})$, in which the magnitude of the velocity varies much widely and randomly (Figures $7(a)$ and $8(e)$ ). When this dynamic arises, the QUAV undergoes excessive wear that may shorten its lifetime and may even be catastrophic. Other dynamics also exist. When $\Omega_{1} \in[203,216] \cup$ $[244,280](\mathrm{rad} / \mathrm{s})$, the system switches to different angular velocities periodically (Figures $7(\mathrm{a})$ and $8(\mathrm{~g})$ ). This remains a big problem with the hovering state.

The reason for this bifurcation of the dynamical behavior is the reaction of the change in torque as the speed of the front propeller, $\Omega_{1}$, changes. To analyze the changes in the four torques is complicated because of their vector nature. Instead, we focus on the scalar quantity energy, especially the Casimir energy and Casimir power. The Casimir power formula (20) is related to the front speed $\Omega_{1}$ and the difference of speed of the front and left propellers $c$. As $\Omega_{1}$ or $c$ varies, the power changes.

The bifurcation diagram of the Casimir power (Figure $7(b)$ ) is identical to the state bifurcation of $p$ 


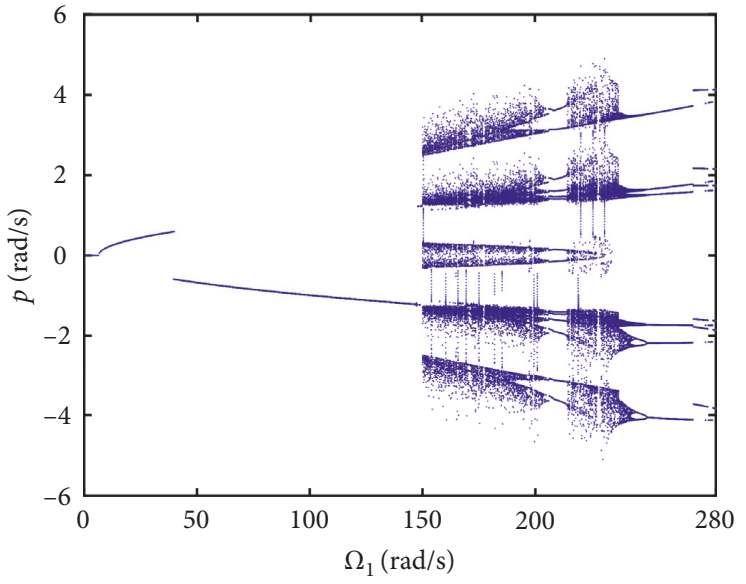

(a)

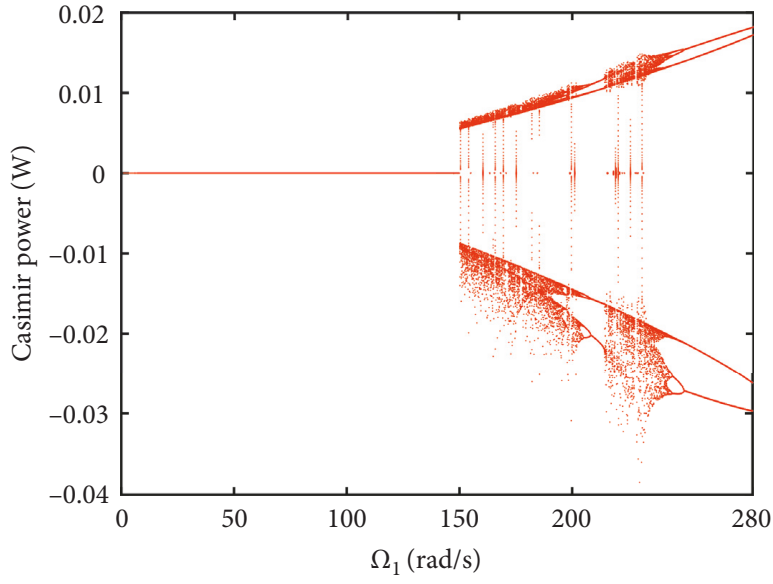

(b)

FIgURe 7: Bifurcation diagrams of (a) $p$ and (b) Casimir power with respect to $\Omega_{1}$ with the initial value of $\boldsymbol{\omega}_{0}=\left[\begin{array}{lll}p_{0} & q_{0} & r_{0}\end{array}\right]^{\mathrm{T}}=$ $\left[\begin{array}{lll}0.001 & 0.001 & 0.001\end{array}\right]^{\mathrm{T}}(\mathrm{rad} / \mathrm{s})$ and $c=5 \mathrm{rad} / \mathrm{s}$.

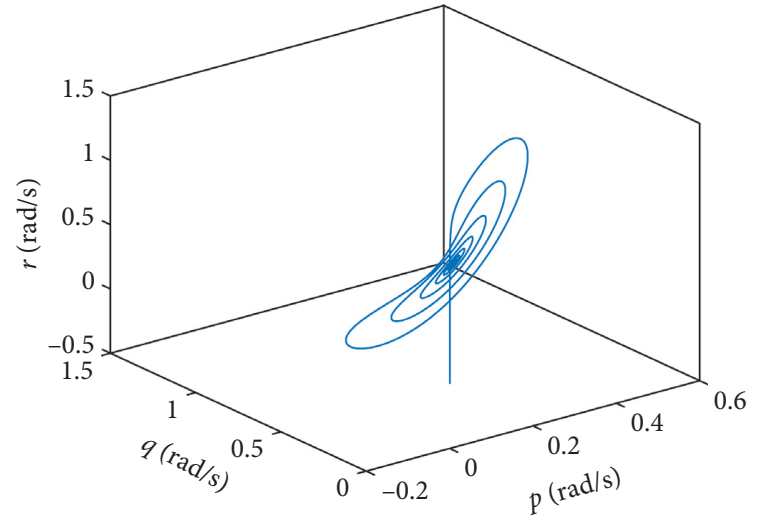

(a)

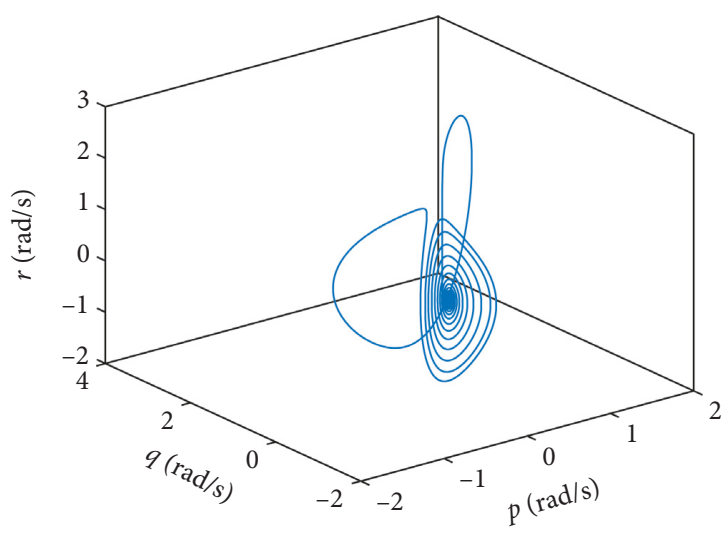

(c)

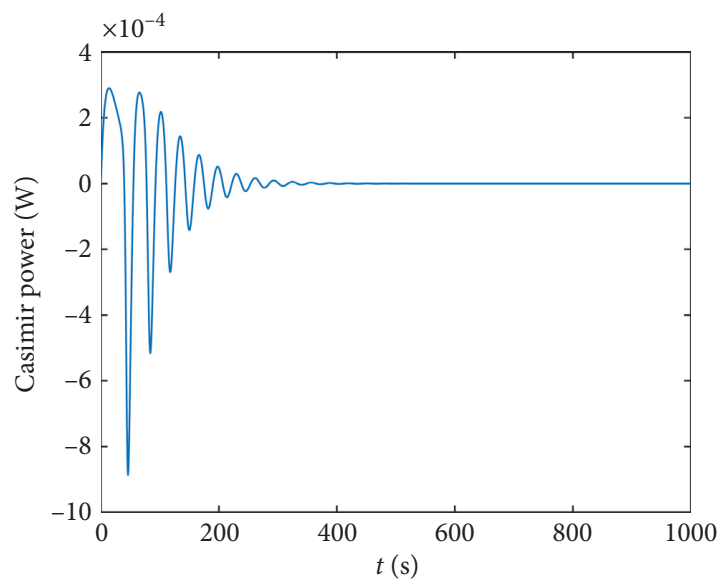

(b)

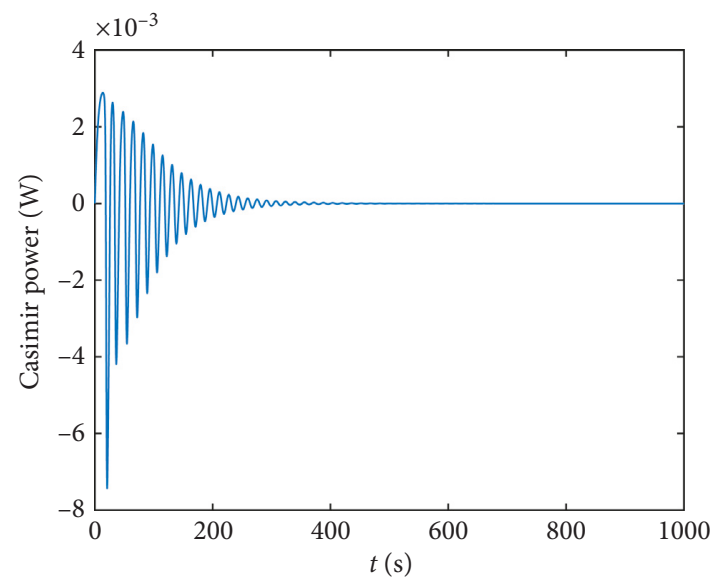

(d)

Figure 8: Continued. 


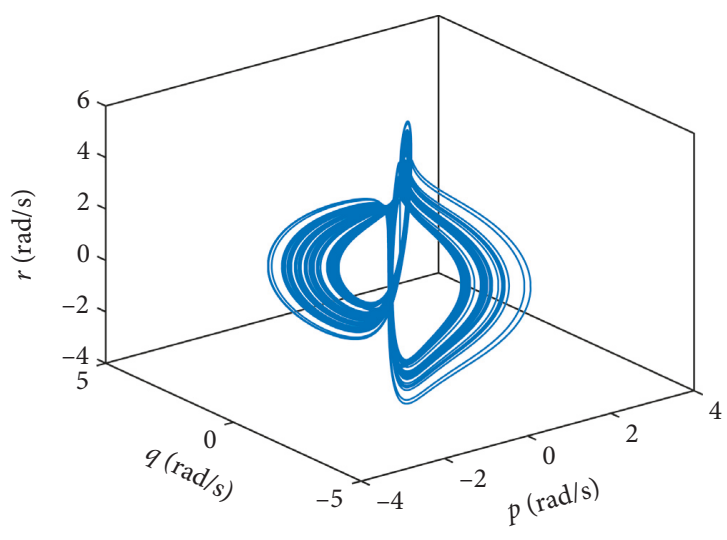

(e)

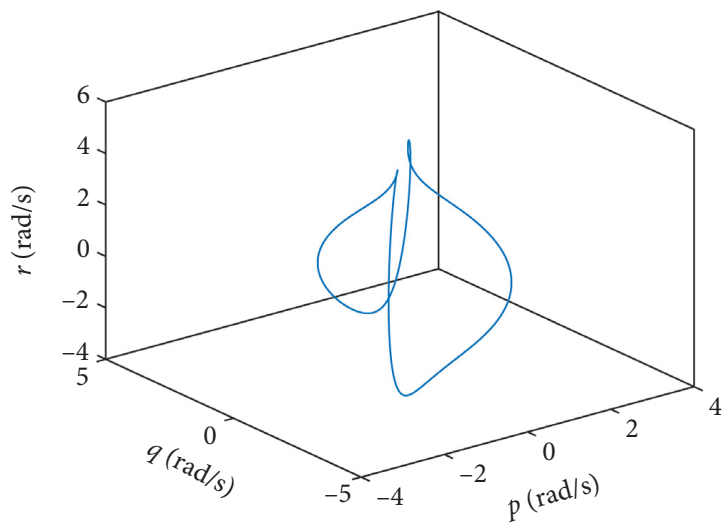

(g)

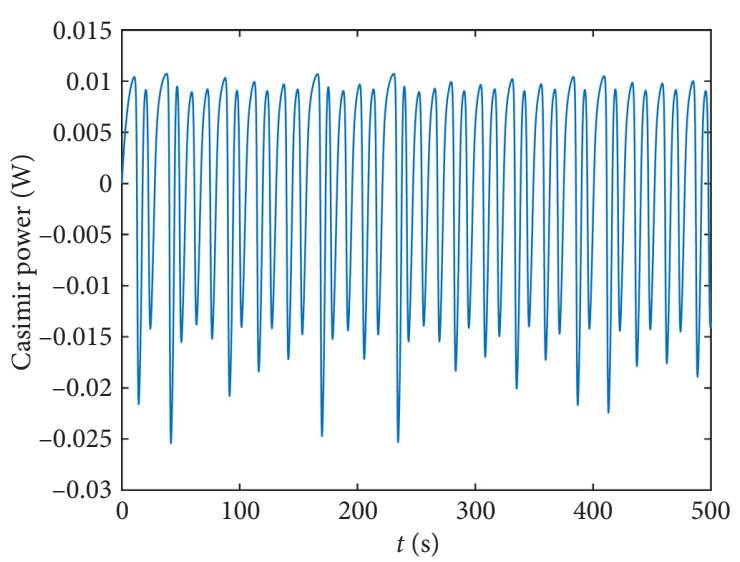

(f)

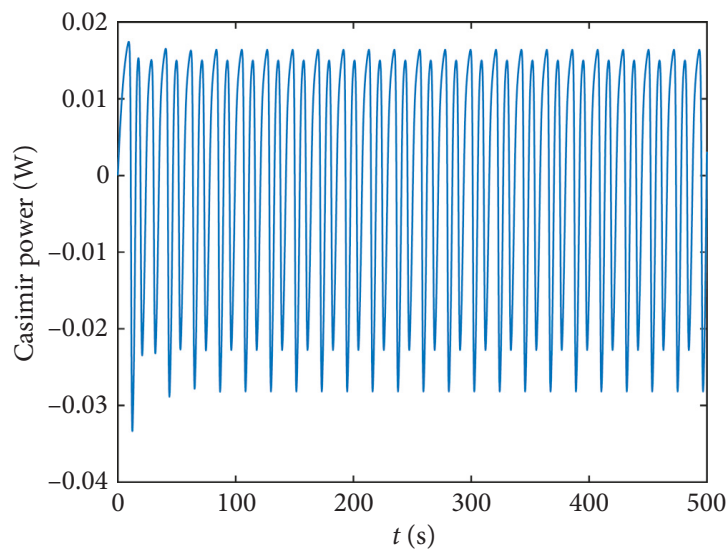

(h)

FIgURE 8: 3D view (left column) and Casimir power (right column) of system (15) with (a, b) $\Omega_{1}=30 \mathrm{rad} / \mathrm{s},(\mathrm{c}, \mathrm{d}) \Omega_{1}=100 \mathrm{rad} / \mathrm{s},(\mathrm{e}, \mathrm{f})$ $\Omega_{1}=196.4 \mathrm{rad} / \mathrm{s}$, and $(\mathrm{g}, \mathrm{h}) \Omega_{1}=260 \mathrm{rad} / \mathrm{s}$.

(Figure $7(\mathrm{a})$ ). When $\Omega_{1} \in(0,147](\mathrm{rad} / \mathrm{s})$, the Casimir power converges to zero. The convergence is clearly demonstrated by the time series of the Casimir power (Figures $8(\mathrm{~b})$ and $8(\mathrm{~d})$ ), which means that, during energy exchange, the supplied power falls gradually below the dissipative power. This allows the dynamical behavior to converge finally to one of two sinks, either $E_{2}$ (Figure 8(b)) or $E_{3}$ (Figure 8(c)). Remark 3(6) points out this conclusion. After the parameter point $\Omega_{1}=147 \mathrm{rad} / \mathrm{s}$, the Casimir power bursts and irregularly switches along the zero line (Figures 7(b) and 8(f)), which means the Casimir energy leaps from one level to another level higher; the energy exchange is sudden and sets up an imbalance between the supplied and the dissipated power. The resultant torque from the propelling thrust and air drag is not in synchrony but conflicts. Consequently, the angular velocity enters a chaotic mode, as described in Remark 3(5). For a periodic orbit, taking $\Omega_{1}=260(\mathrm{rad} / \mathrm{s})$, the Casimir power operates multiperiodically along the zero line (Figure $8(\mathrm{~h})$ ); the velocity of the system also behaves multiperiodically (Figure 8(g)), as claimed in Remark 3(4). The identification between the bifurcation of the Casimir power (Figure 7(b)) and the bifurcation of the angular speed $p$ (Figure 7(a)) clearly demonstrates that the energy exchange is the main mechanism for different dynamics.

When $\Omega_{1}=196.4 \mathrm{rad} / \mathrm{s}$, the system undergoes chaotic behavior (Figure 8(e)), but the chaotic degree is not so strong as that in some classic systems, such as the Lorenz system and the Chen system. This is true in practice because the QUAV system has four rotors and therefore does not normally operate chaotically in most settings. Even if it oscillates chaotically, the degree is not so dramatic. We may find some explanation from the nature of stability of the equilibrium points. In Table 3, we list the equilibrium points and the corresponding eigenvalues of the chaotic system under different parameter values $\Omega_{1}$; the remaining parameters are fixed as given in Table 2. Unlike the classic Lorenz system, both the Chen and Lü systems, with their three saddle equilibrium points, easily produce chaos to some degree. We find that, with $\Omega_{1} \in[6.3852,280](\mathrm{rad} / \mathrm{s})$, the system has one saddle equilibrium point and two stable node-foci (Table 3 ). This stability of the equilibrium points is similar to the stability of the system proposed in reference [40] in which weak chaos was produced.

Similarly, to study the influence of the structural parameters, specifically the moment of inertia $I_{x}$ and the speed 
TABLE 3: Equilibria and eigenvalues of system (15).

\begin{tabular}{lccc}
\hline Parameter of $\Omega_{1}$ & Equilibrium & Eigenvalue & Stability type \\
\hline \multirow{2}{*}{$100(\mathrm{rad} / \mathrm{s})$} & $(0,0,5.1250)^{\mathrm{T}}$ & $1.3534,-0.05,-1.4934$ & Saddle index 1 \\
& $( \pm 0.4229, \pm 0.9938,0.4443)^{\mathrm{T}}$ & $-0.1572,-0.0164 \pm 0.3917 i$ & Stable node-focus \\
\hline \multirow{2}{*}{$196.4(\mathrm{rad} / \mathrm{s})$} & $(0,0,9.9450)^{\mathrm{T}}$ & $2.7276,-0.05,-2.8676$ & Saddle index 1 \\
& $( \pm 0.6025, \pm 1.4159,0.4443)^{\mathrm{T}}$ & $-0.1581,-0.0159 \pm 0.5567 i$ & Stable node-focus \\
\multirow{2}{*}{$260(\mathrm{rad} / \mathrm{s})$} & $(0,0,13.1250)^{\mathrm{T}}$ & $3.6340,-0.05,-3.7740$ & Saddle index 1 \\
& $( \pm 0.6961, \pm 1.6358,0.4443)^{\mathrm{T}}$ & $-0.1620,-0.0140 \pm 0.7172 i$ & Stable node-focus \\
\hline
\end{tabular}

of the front propeller $\Omega_{1}$, we varied $I_{x}$ and $\Omega_{1}$, fixing the remaining parameter values as given in Table 2 . The regions of various dynamical orbits are plotted in Figure 9, with each color having the same meaning as in Figure 5. We realized that chaos is more likely to occur with relatively large values of speed $\Omega_{1}$ with increasing $I_{x}$. The bifurcation plots of $p$ (Figure 10(a)) and the Casimir power (Figure 10(b)) against $\Omega_{1}$ are also displayed with $I_{x}=4 e-3 \mathrm{~kg} \cdot \mathrm{m}^{2}$.

We see that, in the region $I_{x} \in[3 e-5,7 e-5]\left(\mathrm{kg} \cdot \mathrm{m}^{2}\right)$ and $\Omega_{1} \in[50,500](\mathrm{rad} / \mathrm{s})$, when $\Omega_{1}=5 \mathrm{rad} / \mathrm{s}$ and $\Omega_{1}=196.4 \mathrm{rad} / \mathrm{s}$, they satisfy the condition of equation (21), and hence, the rotorcraft is in a hovering state. From Figure 9, we can see that when $I_{x} \in[4.8 e-5,5.5 e-5]\left(\mathrm{kg} \cdot \mathrm{m}^{2}\right)$, the QUAV tends to undergo chaos in the hovering state. Therefore, this also must be taken into account when performing a yaw maneuver while hovering or indeed designing the QUAV controller.

\subsection{Rich Dynamics under Different Structural Parameters.} In this section, we study the influence of the structural parameters of system (15) on the system trajectory and the influence of different trajectories on the dynamics of the position system (6).

The initial values are $\boldsymbol{\omega}_{0}=\left[\begin{array}{lll}p_{0} & q_{0} & r_{0}\end{array}\right]^{\mathrm{T}}=\left[\begin{array}{ll}0.001 & 0.001\end{array}\right.$ $0.001]^{\mathrm{T}}(\mathrm{rad} / \mathrm{s}), c=5 \mathrm{rad} / \mathrm{s}$, and $\Omega_{1}=196.4 \mathrm{rad} / \mathrm{s}$, with all other parameter values fixed as given in Table 2, except for the moments of inertia. We still assume that the initial state of the QUAV is hovering. We introduce three different inertial configurations:

Case 1: let $I_{x}=4.9 e-3 \mathrm{~kg} \cdot \mathrm{m}^{2}, I_{y}=9.8 e-3 \mathrm{~kg} \cdot \mathrm{m}^{2}$, $I_{z}=8.8 e-3 \mathrm{~kg} \cdot \mathrm{m}^{2}$, and $I_{r}=3.4 e-5 \mathrm{~kg} \cdot \mathrm{m}^{2}$, then the system (15) undergoes chaos (Figure 11(a)).

Case 2: let $I_{x}=4.9 e-3 \mathrm{~kg} \cdot \mathrm{m}^{2}, I_{y}=9.8 e-3 \mathrm{~kg} \cdot \mathrm{m}^{2}$, $I_{z}=8.8 e-3 \mathrm{~kg} \cdot \mathrm{m}^{2}$, and $I_{r}=3.4 e-4 \mathrm{~kg} \cdot \mathrm{m}^{2}$, then the orbit of system (15) is periodic (Figure 11(b)).

Case 3: let $I_{x}=6.8 e-3 \mathrm{~kg} \cdot \mathrm{m}^{2}, I_{y}=7.8 e-3 \mathrm{~kg} \cdot \mathrm{m}^{2}$, $I_{z}=8.8 e-3 \mathrm{~kg} \cdot \mathrm{m}^{2}$, and $I_{r}=3.4 e-5 \mathrm{~kg} \cdot \mathrm{m}^{2}$, then the trajectory converges to a sink (Figure 11(c)).

When evolving from Case 1 to Case 2, the trajectory of the system transitions from chaotic to periodic behavior when only the moment of inertia $I_{r}$ is changed. This change can be obtained simply by changing the radius of the rotors but without changing the overall weight of the QUAV. When evolving from Case 1 to Case 3, we similarly keep the values of all other parameters unchanged except for the

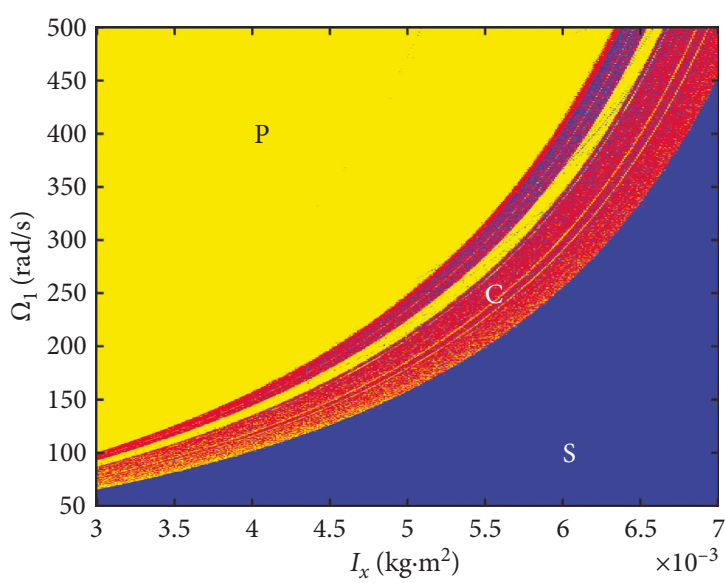

Figure 9: Three colored dynamical regions of system (15) at the initial value of $\omega_{0}=\left[\begin{array}{lll}p_{0} & q_{0} & r_{0}\end{array}\right]^{\mathrm{T}}=\left[\begin{array}{lll}0.001 & 0.001 & 0.001\end{array}\right]^{\mathrm{T}}(\mathrm{rad} / \mathrm{s})$ with $I_{x} \in[3 e-3,7 e-3]\left(\mathrm{kg} \cdot \mathrm{m}^{2}\right)$ and $\Omega_{1} \in[50,500](\mathrm{rad} / \mathrm{s})$.

moments of inertia $I_{x}$ and $I_{y}$. This is achieved by adjusting the weight ratio or the shape of the rotor along the $x$ - and $y$-axes. Then, the initial chaotic trajectory converges to a sink.

Note that the main variable in system (15) is the QUAV's angular velocity. As a consequence, various dynamical behaviors exhibited by the QUAV are nearly imperceptible, and we observe the physical Euler angles in the attitude system. The chaotic behavior of angular velocity is not necessarily chaotic in the Euler angles. This is why chaotic behavior is not observed by researchers, and hence, the phenomenon and its characteristics have been ignored. The relationship between the angular velocities of the three axes in the B-frame and the angular speeds in the E-frame (inertial frame) is expressed in equation (16).

Consider the resultant Euler angle after the transformation of equation (16) for these three cases. The blue, orange, and green curves in Figure 12 correspond to the trajectories for Cases 1,2, and 3, respectively. The time series of the angular velocity $p$ in Case 1 indicates chaos (Figure 12(a)). The oscillation amplitude of the angular velocity $p$ is quite large. Note that, at this time, we are performing the yaw maneuver, so this large oscillation in angular velocity $p$ is undesired. For Case 2, the orbit of angular velocity is periodic for which the amplitude is apparently smaller than that for Case 1 . For Case 3, where the angular velocity $p$ becomes attracted to a sink, its value is almost equal to zero and the QUAV is relatively stable and easy to control. 


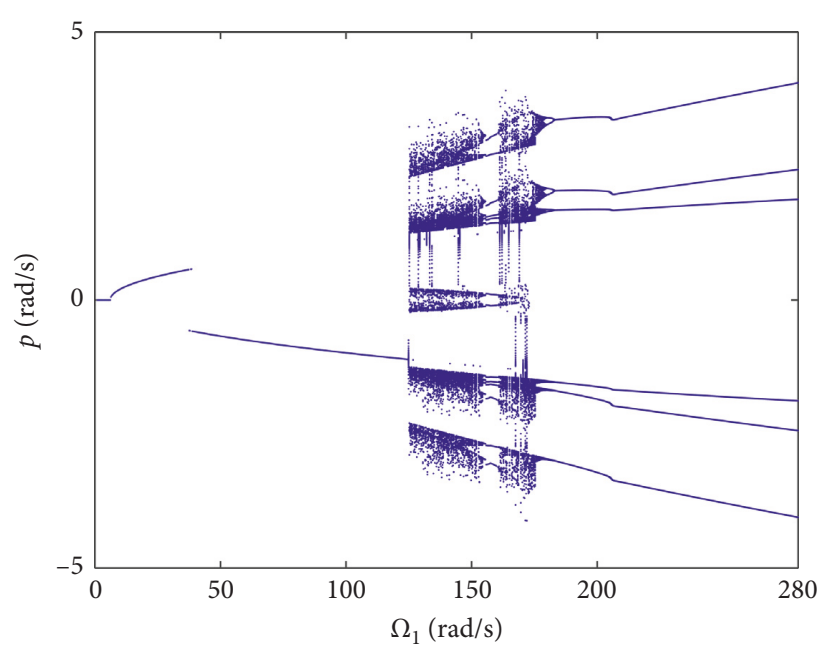

(a)

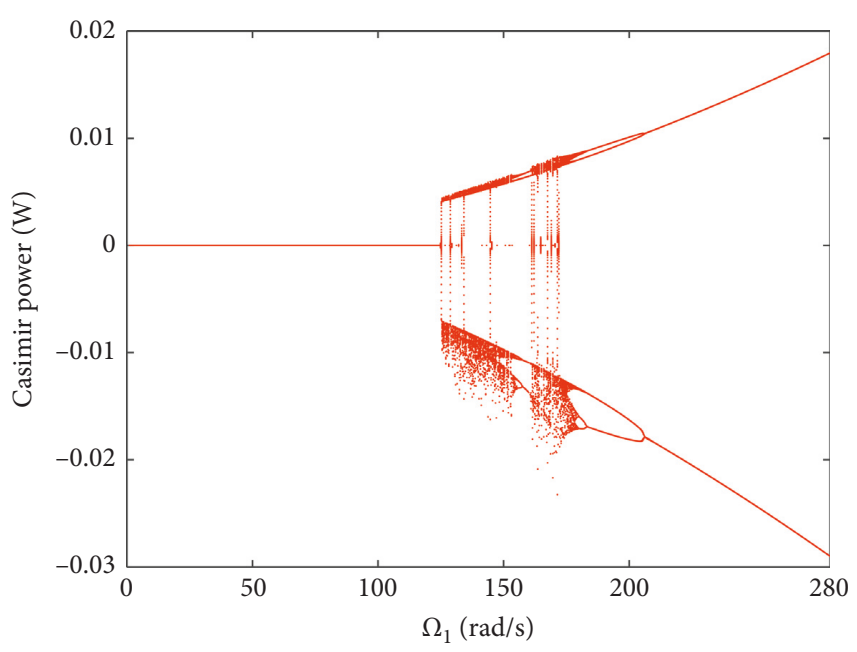

(b)

FIgure 10: Bifurcation diagrams of (a) $p$ and (b) Casimir power with respect to $\Omega_{1}$ with the initial value of $\boldsymbol{\omega}_{0}=\left[\begin{array}{lll}p_{0} & q_{0} & r_{0}\end{array}\right]^{\mathrm{T}}=$ $\left[\begin{array}{lll}0.001 & 0.001 & 0.001\end{array}\right]^{\mathrm{T}}(\mathrm{rad} / \mathrm{s})$ and $I_{x}=4 e-3 \mathrm{~kg} \cdot \mathrm{m}^{2}$.

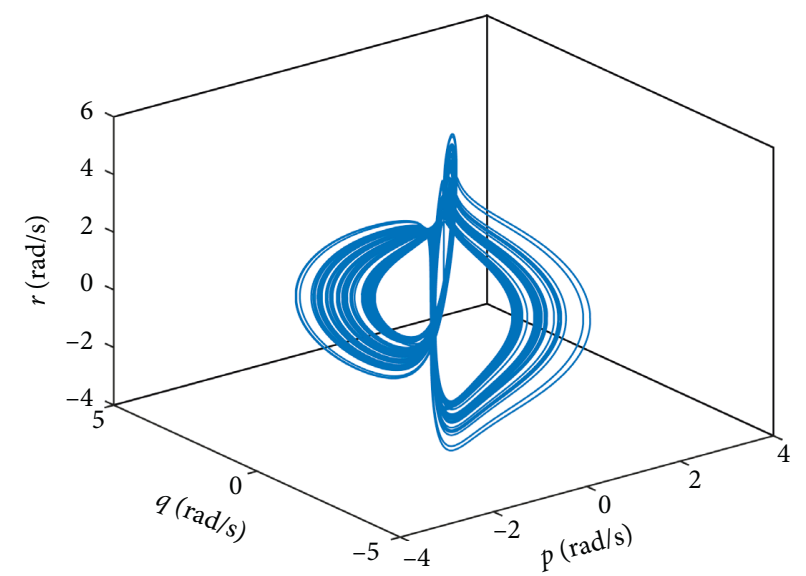

(a)

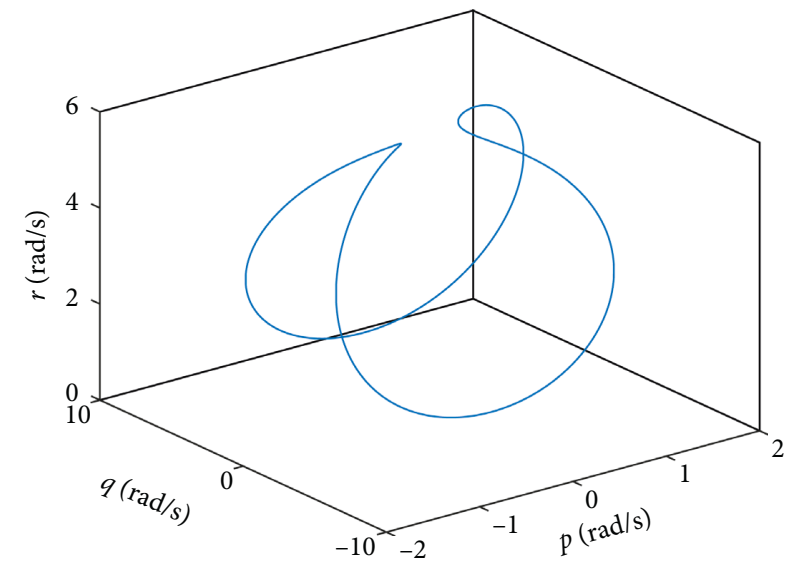

(b)

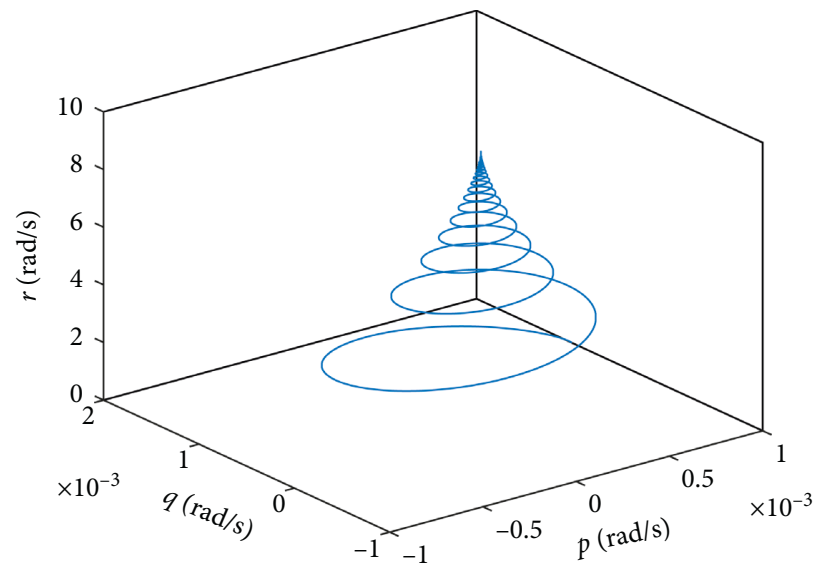

(c)

FIGURE 11: Trajectories obtained from (a) case 1: chaos, (b) case 2: periodicity, and (c) case 3: sink.

In Figure 12(b), in the chaotic state for angular velocity (Case 1), the roll angle $\phi$ does not behave so erratically as the angular velocity, $p$; however, we still find that the roll angle reaches $2.5546 \mathrm{rad}=146.95^{\circ}$ after $15 \mathrm{~s}$, indicating the maneuver is dangerous and a likelihood the QUAV will crash. For the periodic orbit for angular velocity (Case 2), the roll 

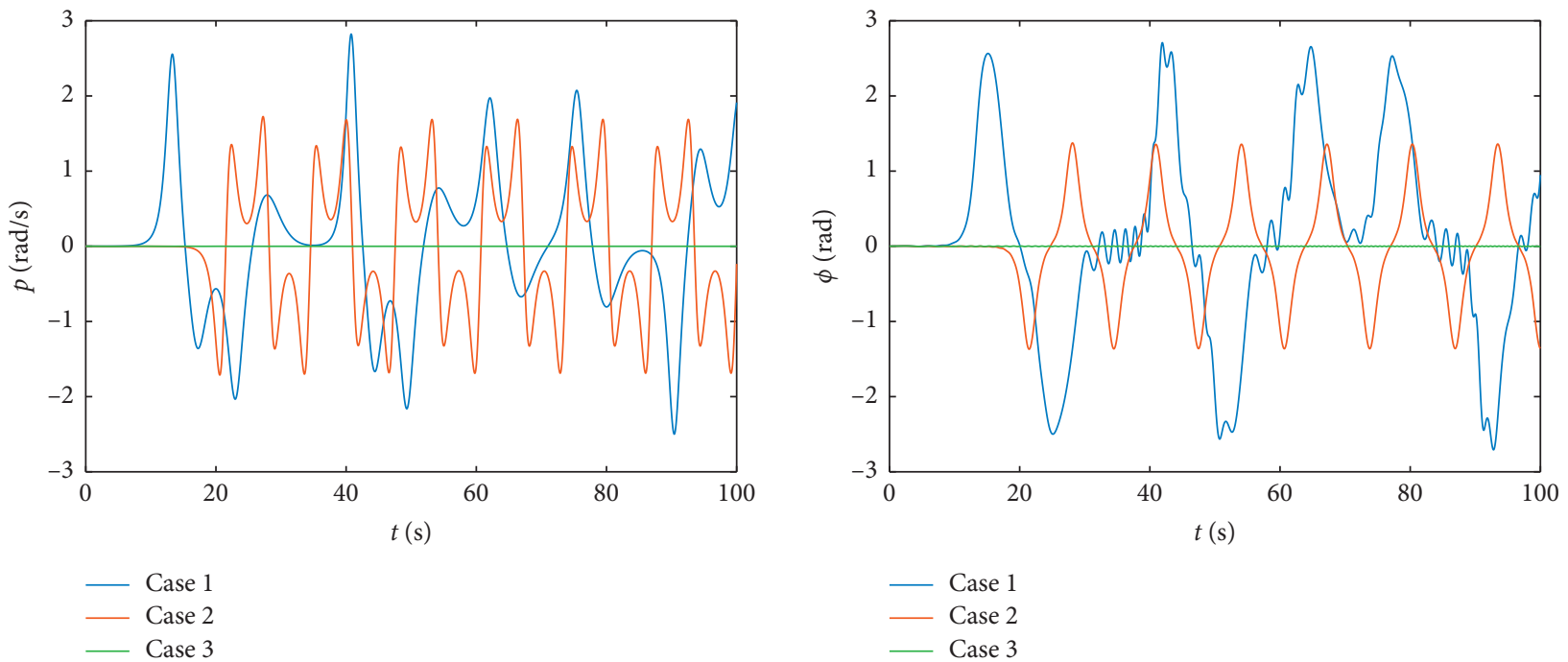

(a)

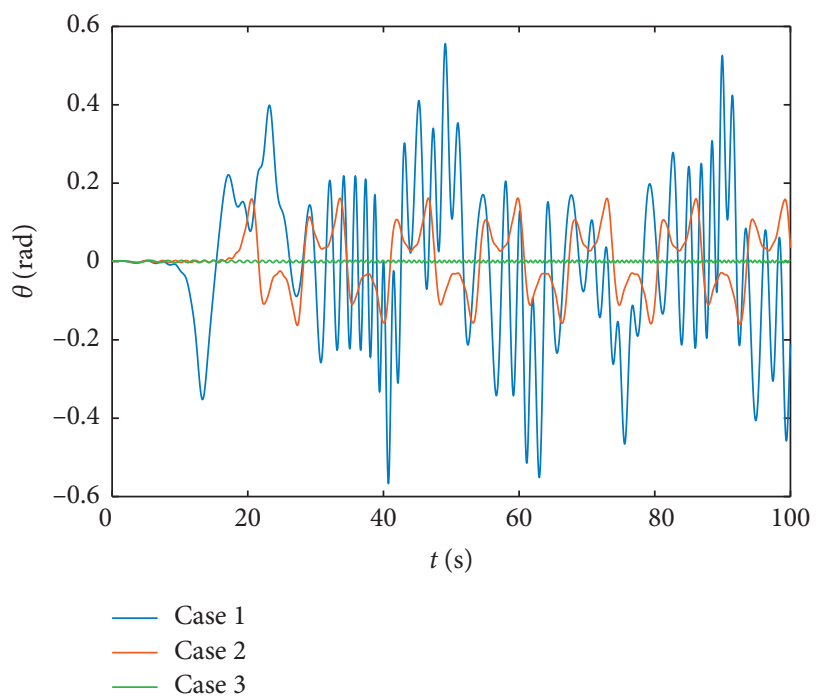

(b)

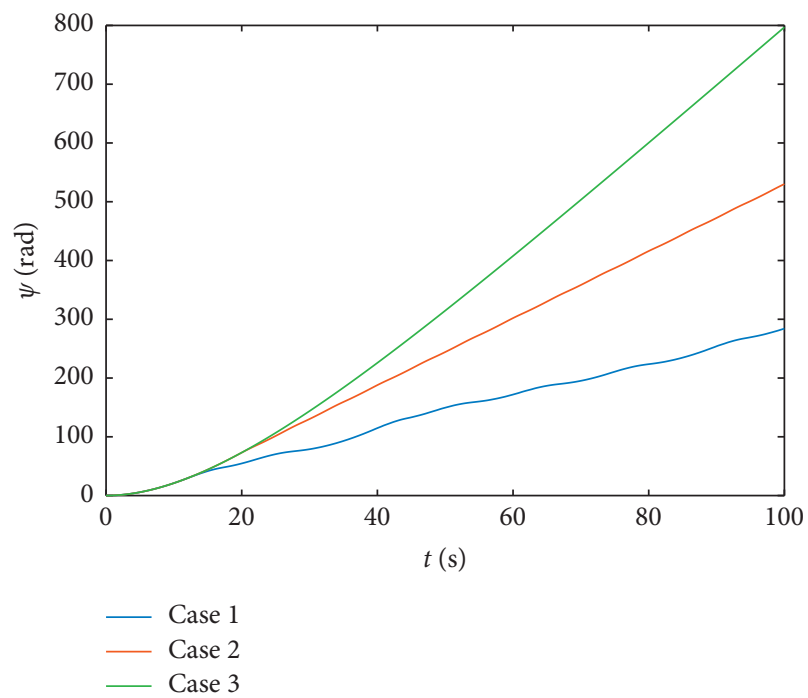

(c)

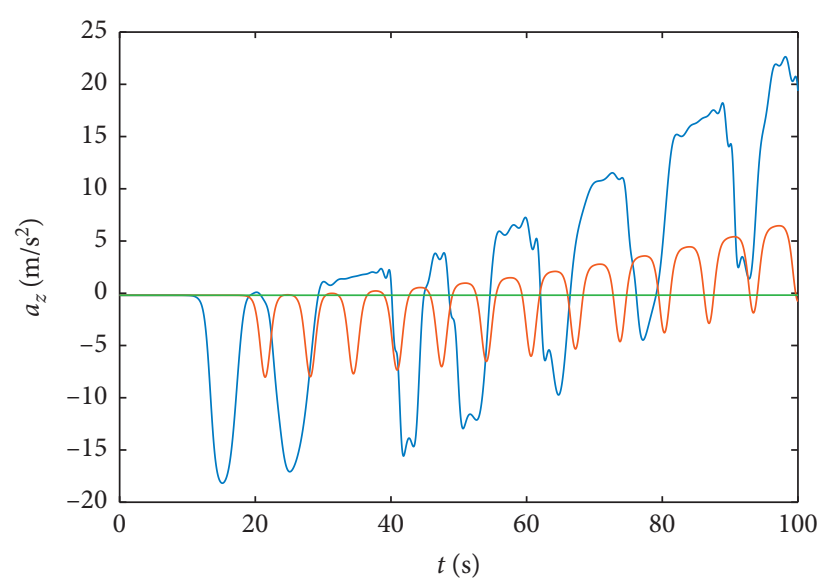

(d)

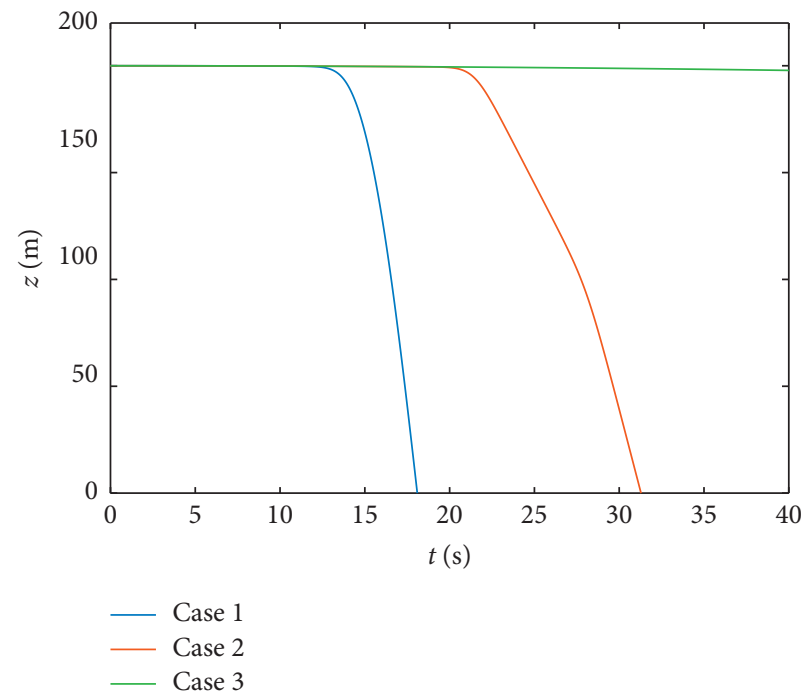

(e)

(f)

FIGURE 12: Time series of QUAV characteristics for three different configurations: (a) angular velocity $p$; (b) roll angle $\phi$; (c) pitch angle $\theta$; (d) yaw angle $\psi$; (e) acceleration along the $z$-axis; (f) displacement along the $z$-axis. 
angle also behaves periodically. The roll angle peaks at $-1.3689 \mathrm{rad}=-78.4^{\circ}$ after $22 \mathrm{~s}$. In addition, we observed that, for Cases 1 and 2, the maximum amplitude of the roll angle is larger than that of the pitch angle $\theta$ (Figure 12(c)). This dynamical feature arises because $I_{x}$ is much smaller than $I_{y}$. In addition, comparing these two cases, the stability of the system is improved when $I_{r}$ is increased. Therefore, we also find that the stability of the system may be optimized by changing $I_{r}$ even if the difference between $I_{x}$ and $I_{y}$ is relatively large. Changing $I_{r}$ is attained by adjusting the radius of the rotor, which is much easier than changing the overall structures of the QUAV. All of these factors should be considered in the design of the QUAV structure and controller. For Case 3, trajectories of the roll and pitch angles of the QUAV are relatively stable. Moreover, from Figure 12(d), it can be seen that the yaw angle $\psi$ varies the most, and a change in yaw angle is what we expect. Once again, for yaw maneuvering, structural parameters have been demonstrated to be superior for Case 3 that for the other two cases.

From the first equation of equation (6), we find the acceleration of the QUAV along the $z$-axis to be

$$
a_{z}=\ddot{z}=\frac{\left(F_{T} \cos \phi \cos \theta-k_{3} \dot{z}-m g\right)}{m} .
$$

For Case 1, the QUAV has an acceleration $a_{z}$ of $-18.2 \mathrm{~m} / \mathrm{s}^{2}$ after $15 \mathrm{~s}$ (Figure 12(e)), the acceleration being almost twice that of the acceleration of gravity. This further indicates that when chaos takes place, the QUAV is in a precarious region and descends very rapidly (Figure 12(f)). If the QUAV flies at an altitude of $200 \mathrm{~m}$, it falls to the ground in only 17.66 s. In Case 2 , the acceleration of the QUAV $a_{z}$ reaches $-8.0272 \mathrm{~m} / \mathrm{s}^{2}$ after $22 \mathrm{~s}$ but still rapidly descends (Figure 12(f)). However, the decline for Case 1 is much slower than that for Case 2, falling to the ground in about $32 \mathrm{~s}$ from the same altitude (Figure 12(f)). For Case 3, the change in $a_{z}$ is very small at this time; consequently, the flight of the QUAV is relatively stable.

The summary of the section is stated as follows.

The rich dynamics of the system uncovered mainly via two bifurcation regions reveal the effects of different inputs on the dynamics of the system under fixed structural parameters and uncover the impact of active input and structural parameters on system stability. The coincidence between the state bifurcation and Casimir bifurcation demonstrates that the change in Casimir power reflects the dynamic properties of the system. The importance of selecting suitable structural parameters that improve the stability of the rotorcraft has been demonstrated via a comparison of the three cases. Specifically, the rotorcraft is much easier to control when the trajectory of the QUAV attitude system converges to a sink rather than it goes into chaotic or periodic states. The dynamic analysis in this section has important guidance in designing the shape, the bobweight of a QUAV, the driving force direction, and the magnitude. All of these help to avoid producing large oscillations in speed and even generating chaos due to incorrect configuration parameters or precipitous driving forces. Moreover, the detailed dynamic analysis guides the design of the controller to attain an anticipated performance. If a strong controller that takes no account of the characteristics of the open-loop QUAV is designed, the control has to consume the energy in suppressing the oscillations. A strong controller would also destroy the actuator through the crude application of forces.

\section{Boundary of the QUAV Attitude System}

The extremal points of the Casimir function of the QUAV attitude system are determined by setting $\dot{C}=0$ in equation (20); the triaxle ellipsoid equation then becomes

$$
\Xi_{e}: k_{4} p^{2}+k_{5} q^{2}+k_{6} r-\left(\frac{d\left(c^{2}+2 c \Omega_{1}\right)}{k_{6}}\right)^{2}=d^{2} \frac{c^{2}+2 c \Omega_{1}^{2}}{k_{6}} .
$$

For $\forall \boldsymbol{\omega}=[p, q, r]^{\mathrm{T}} \neq 0$, when the trajectory of the system runs inside $\Xi_{e}, \dot{C}>0$, the Casimir energy increases until $\dot{C}=0$ (red "O" in Figures 13(a) and 13(b)), where the maxima are obtained. Conversely, for $\forall \boldsymbol{\omega} \neq 0$, when the trajectory of the system runs outside $\Xi_{\mathrm{e}}, \dot{C}<0$, the Casimir energy decreases until $\dot{C}=0$ (yellow “ $*$ " in Figures 13(a) and 13(b)), where the minima are obtained. To see the maxima and minima more clearly, we removed the ellipsoid in Figure 13(b). In Figures 13(a) and 13(b), green " $\triangle$ " and “ $\lesssim$ " are the equilibrium points of the system, which must be on the surface of $\Xi_{\mathrm{e}}$ because $\dot{C}=(\partial C / \partial \boldsymbol{\omega}) \dot{\boldsymbol{\omega}}=0$ when $\dot{\boldsymbol{\omega}}=0$.

Here, via the Lagrange multiplier method, we simply give the supremum bound of system (15).

Theorem 1. The QUAV attitude chaotic attractor has supremum ellipsoid:

$$
\Xi_{s}: I_{x} p^{2}+I_{y} q^{2}+I_{z} r^{2}=\frac{4 d^{2} I_{z}\left(c^{2}+2 c \Omega_{1}\right)^{2}}{k_{6}^{2}} .
$$

Proof. The supremum bound is defined as

$$
\begin{aligned}
& \max \quad C=\frac{1}{2}\left(I_{x} p^{2}+I_{y} q^{2}+I_{z} r^{2}\right) . \\
& \text { s.t. } \quad \dot{C}=0
\end{aligned}
$$

and therefore,

$$
\left(k_{4} p^{2}+k_{5} q^{2}+k_{6} r^{2}\right)=2 d\left(c^{2}+2 c \Omega_{1}\right) r .
$$

The Lagrange function becomes

$$
\begin{aligned}
L= & \frac{1}{2}\left(I_{x} p^{2}+I_{y} q^{2}+I_{z} r^{2}\right) \\
& +\lambda\left[k_{4} p^{2}+k_{5} q^{2}+k_{6} r^{2}-2 d\left(c^{2}+2 c \Omega_{1}\right) r\right],
\end{aligned}
$$

where $\lambda$ is the Lagrange multiplier. The derivatives of $L$ for each of its variables are 


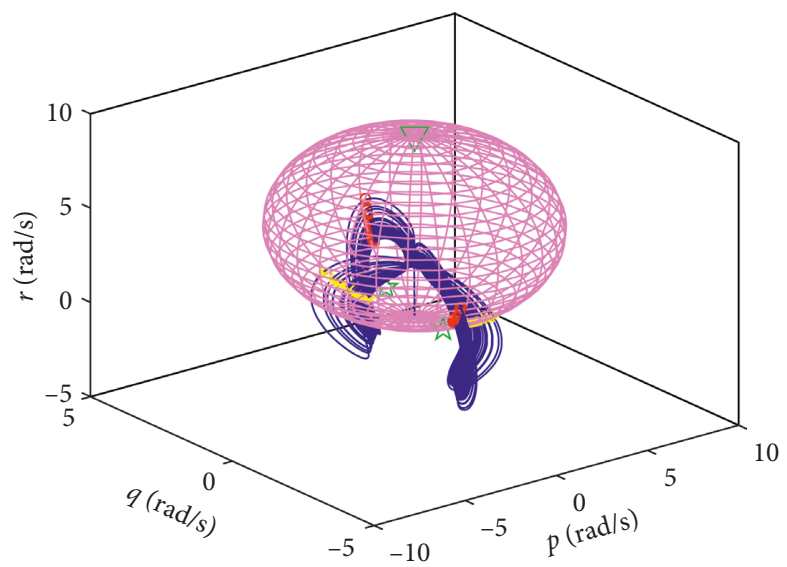

(a)

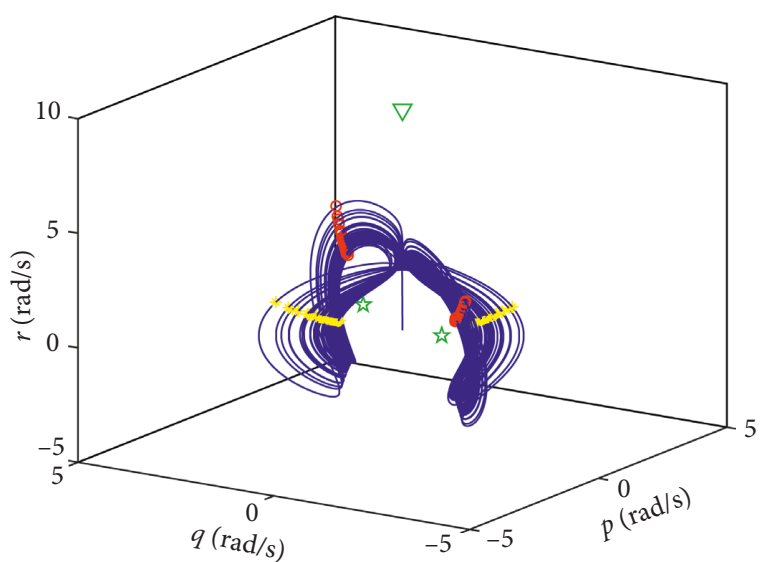

(b)

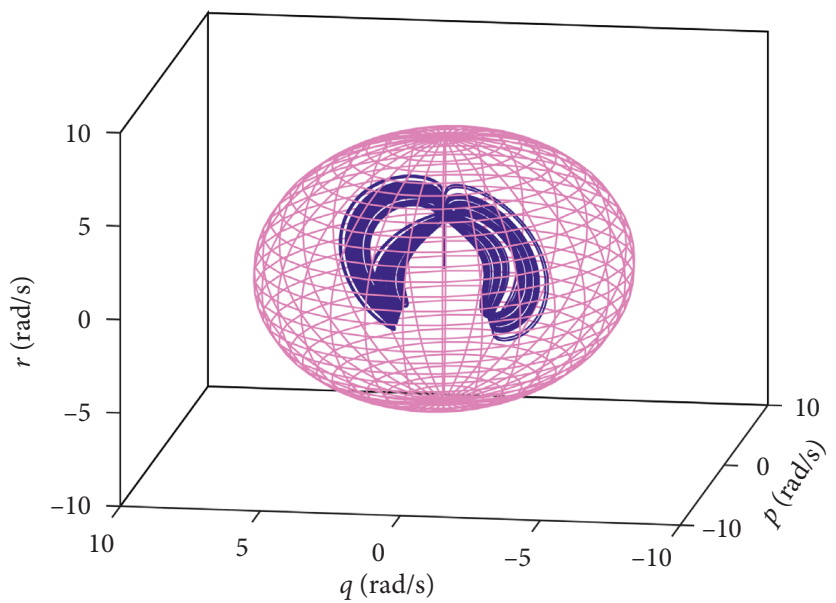

(c)

FIGURE 13: System analysis via the Casimir function and Casimir power: (a) intersection points of the invariant ellipsoid and chaotic attractor with three equilibrium points; (b) intersection points with the invariant ellipsoid omitted; (c) the supremum ellipsoid of the QUAV attitude chaotic attractor.

$$
\left\{\begin{array}{l}
\frac{\partial L}{\partial p}=I_{x} p+2 k_{4} p \lambda=0, \\
\frac{\partial L}{\partial q}=I_{y} q+2 k_{5} q \lambda=0, \\
\frac{\partial L}{\partial r}=I_{z} r+\lambda\left[2 k_{6} r-2 d\left(c^{2}+2 c \Omega_{1}\right)\right]=0 \\
\frac{\partial L}{\partial \lambda}=k_{4} p^{2}+k_{5} q^{2}+k_{6} r^{2}-2 d\left(c^{2}+2 c \Omega_{1}\right) r=0
\end{array}\right.
$$

We identify two stagnation points: $p=0(\mathrm{rad} / \mathrm{s})$, $q=0(\mathrm{rad} / \mathrm{s}), r=0(\mathrm{rad} / \mathrm{s})$ and $p=0(\mathrm{rad} / \mathrm{s}), q=0(\mathrm{rad} / \mathrm{s})$, $r=2 d\left(c^{2}+2 c \Omega_{1}\right) / k_{6}(\mathrm{rad} / \mathrm{s})$. Clearly, the maximum value is obtained with the latter, with the supremum ellipsoid being

$$
\Xi_{s}: I_{x} p^{2}+I_{y} q^{2}+I_{z} r^{2}=\frac{4 d^{2} I_{z}\left(c^{2}+2 c \Omega_{1}\right)^{2}}{k_{6}^{2}},
$$

which is illustrated in Figure 13(c). The proof of Theorem 1 is completed.
The supremum bound of the QUAV attitude system (15) was provided in this section. If the trajectory of a system continuously shrinks, it converges onto a sink. If the system trajectory expands unboundedly, it acts as a source and no chaos emerges. For a chaotic system, there exists a positive LE making the system grow exponentially. However, if the orbits are confined within a bound, the system expands and contracts continuously in a dimension of fractional number with zero phase space. Therefore, the boundary to the system trajectory is a necessary condition in generating chaos. In addition, the boundary also implies that the system's total energy and amplitude of the variable cannot grow unboundedly. The global stability of the strange attractor is formed because of the boundary.

\section{Conclusion}

A model of a chaotic QUAV attitude rigid-body system was established. The pitchfork bifurcation of the equilibrium points was displayed revealing the hidden trouble in the QUAV dynamics. The rich dynamics of the system were specified via the regions of various dynamical behaviors and 
bifurcations of the structural and rotational speed parameters of the system. The regions in which the rotor speeds and structural parameters tended towards chaotic behavior in the hovering state were analyzed in detail. Furthermore, the dynamics of the system were verified through the Casimir power bifurcation. The stability of the system is enhanced by optimizing the structural parameters, which we illustrated through three cases with different structural parameters. Finally, the supremum bound of the chaotic attractor was presented using the Casimir power and the Lagrange multiplier method. For the chaotic model of the QUAV, there is a large parameter space that needs to be investigated in terms of control, the fractional-order system, dynamic analysis, hidden chaos, and transient chaos. How to design the controller when the system enters into the parameter region of chaos or oscillation is a challengeable task. The hidden chaos and multistability are also the open topics for other researchers.

\section{Data Availability}

The data used to support the findings of this study are available from the corresponding author upon request.

\section{Conflicts of Interest}

The authors declare no conflicts of interest.

\section{Acknowledgments}

This work was supported by the National Natural Science Foundation of China (61873186), the Tianjin Natural Science Foundation (17JCZDJC38300), and the College Outstanding Youth Talent Support Program Project (GXYQ2017014). The authors thank Richard Haase, Ph.D., from Liwen Bianji, Edanz Group China (http://www. liwenbianji.cn/ac) for editing the English text of the draft of this manuscript.

\section{References}

[1] E.-H. Zheng, J.-J. Xiong, and J.-L. Luo, "Second order sliding mode control for a quadrotor UAV," ISA Transactions, vol. 53, no. 4, pp. 1350-1356, 2014.

[2] L. Derafa, A. Benallegue, and L. Fridman, "Super twisting control algorithm for the attitude tracking of a four rotors UAV," Journal of the Franklin Institute, vol. 349, no. 2, pp. 685-699, 2012.

[3] A. Taybei and S. Mcgivray, "Attitude stabilization of a VTOL quadrotor aircraft," IEEE Transactions on Control Systems Technology, vol. 14, no. 3, pp. 562-571, 2006.

[4] H. Shim and Y.-J. Joo, "State space analysis of disturbance observer and a robust stability condition," in Proceeding of the IEEE Conference on Decision and Control, New Orleans, LA, USA, December 2007.

[5] A. L. Salih, M. Moghavvemi, H. A. F. Mohamed, and K. S. Gaeid, "Modelling and PID controller design for a quadrotor unmanned air vehicle," in Proceeding of the IEEE International Conference on Automation Quality and Testing Robotics, Cluj-Napoca, Rumania, 2010.
[6] G. M. Hoffmann, D. G. Rajnarayan, S. L. Waslander, and D. Dostal, "The stanford test bed of autonomous rotorcraft for multi agent control," in Proceeding of the Digital Avionics Systems Conference, Salt Lake City, UT, USA, October 2004.

[7] Y. Yuan, L. Cheng, Z. Wang, and C. Sun, "Position tracking and attitude control for quadrotors via active disturbance rejection control method," Science China Information Sciences, vol. 62, no. 1, p. 10201, 2019.

[8] M. Tarhan and E. Altuğ, "EKF based attitude estimation and stabilization of a quadrotor UAV using vanishing points in catadioptric images," Journal of Intelligent and Robotic Systems, vol. 62, no. 3-4, pp. 587-607, 2011.

[9] B. Tian, J. Cui, H. Lu, Z. Zuo, and Q. Zong, "Adaptive finitetime attitude tracking of quadrotors with experiments and comparisons," IEEE Transactions on Industrial Electronics, vol. 66, no. 12, pp. 9428-9438, 2019.

[10] L. Zhao, Y. Jia, and J. Yu, "Adaptive finite-time bipartite consensus for second-order multi-agent systems with antagonistic interactions," Systems \& Control Letters, vol. 102, pp. 22-31, 2017.

[11] F. Chen, R. Jiang, K. Zhang, B. Jiang, and G. Tao, "Robust backstepping sliding-mode control and observer-based fault estimation for a quadrotor UAV," IEEE Transactions on Industrial Electronics, vol. 63, no. 8, pp. 5044-5056, 2016.

[12] H. Wang, X. Ye, Y. Tian, G. Zheng, and N. Christov, "Modelfree-based terminal SMC of quadrotor attitude and position," IEEE Transactions on Aerospace and Electronic Systems, vol. 52, no. 5, pp. 2519-2528, 2016.

[13] B. Mu, K. Zhang, and Y. Shi, "Integral sliding mode flight controller design for a quadrotor and the application in a heterogeneous multi-agent system," IEEE Transactions on Industrial Electronics, vol. 64, no. 12, pp. 9389-9398, 2017.

[14] O. Mofid and S. Mobayen, "Adaptive sliding mode control for finite-time stability of quad-rotor UAVs with parametric uncertainties," ISA Transactions, vol. 72, pp. 1-14, 2018.

[15] J.-J. Xiong and E.-H. Zheng, "Position and attitude tracking control for a quadrotor UAV," ISA Transactions, vol. 53, no. 3 , pp. 725-731, 2014.

[16] A. Modirrousta and M. Khodabandeh, "A novel nonlinear hybrid controller design for an uncertain quadrotor with disturbances," Aerospace Science and Technology, vol. 45, pp. 294-308, 2015.

[17] T. S. Kim, K. Stol, and V. Kecman, "Control of 3 DOF quadrotor model," Lecture Notes in Control and Information Sciences, vol. 360, pp. 29-38, 2007.

[18] H. Voos, "Nonlinear control of a quadrotor micro-UAV using feedback-linearization," in Proceeding of the IEEE International Conference on Mechatronics, Malaga, Spain, August 2009.

[19] J. Kuang, S. H. Tan, and A. Y. T. Leung, "Chaotic attitude motion of satellites under small perturbation torques," Journal of Sound and Vibration, vol. 235, no. 2, pp. 175-200, 2000.

[20] M. Faramin and M. Ataei, "Chaotic attitude analysis of a satellite via Lyapunov exponents and its robust nonlinear control subject to disturbances and uncertainties," Nonlinear Dynamics, vol. 83, no. 1-2, pp. 361-374, 2016.

[21] A. V. Doroshin, "Modeling of chaotic motion of gyrostats in resistant environment on the base of dynamical systems with strange attractors," Communications in Nonlinear Science and Numerical Simulation, vol. 16, no. 8, pp. 3188-3202, 2011.

[22] N. Hemati, "Strange attractors in brushless DC motors," IEEE Transactions on Circuits and Systems I: Fundamental Theory and Applications, vol. 41, no. 1, pp. 40-45, 1994. 
[23] Z. Li, J. B. Park, Y. H. Joo, B. Zhang, and G. Chen, "Bifurcations and chaos in a permanent- magnet synchronous motor," IEEE Transactions on Circuits and Systems I, vol. 49, no. 3, pp. 383-387, 2002.

[24] G. Qi, "Energy cycle of brushless DC motor chaotic system," Applied Mathematical Modelling, vol. 51, pp. 686-697, 2017.

[25] G. Qi and J. Hu, "Force analysis and energy operation of chaotic system of permanent-magnet synchronous motor," International Journal of Bifurcation and Chaos, vol. 27, no. 14, Article ID 1750216, 2017.

[26] V. Pelino, F. Maimone, and A. Pasini, "Energy cycle for the Lorenz attractor," Chaos, Solitons \& Fractals, vol. 64, pp. 67-77, 2014.

[27] G. Qi and X. Liang, "Mechanism and energy cycling of the Qi four-wing chaotic system," International Journal of Bifurcation and Chaos, vol. 27, no. 12, Article ID 1750180, 2017.

[28] G. Qi and J. Zhang, "Energy cycle and bound of Qi chaotic system," Chaos, Solitons \& Fractals, vol. 99, pp. 7-15, 2017.

[29] X. Liang and G. Qi, "Mechanical analysis of chen chaotic system," Chaos, Solitons and Fractals, vol. 98, pp. 173-177, 2017.

[30] X. Liang and G. Qi, "Mechanical analysis and energy cycle of chen chaotic system," Brazilian Journal of Physics, vol. 47, no. 3, pp. 288-294, 2017.

[31] W. Zhang, Q. L. Wu, M. H. Yao, and E. H. Dowell, “Analysis on global and chaotic dynamics of nonlinear wave equations for truss core sandwich plate," Nonlinear Dynamics, vol. 94, no. 1, pp. 1-17, 2018.

[32] Q. L. Wu, W. Zhang, and E. H. Dowell, "Detecting multi-pulse chaotic dynamics of high-dimensional non-autonomous nonlinear system for circular mesh antenna," International Journal of Non-linear Mechanics, vol. 102, pp. 25-40, 2018.

[33] W. Zhang, Q. L. Wu, and W. S. Ma, "Chaotic wave motions and chaotic dynamic responses of piezoelectric laminated composite rectangular thin plate under combined transverse and in-plane excitations," International Journal of Applied Mechanics, vol. 10, no. 10, Article ID 1850114, 2018.

[34] Q. Wu and G. Qi, "Global dynamics of a pipe conveying pulsating fluid in primary parametrical resonance: analytical and numerical results from the nonlinear wave equation," Physics Letters A, vol. 383, no. 14, pp. 1555-1562, 2019.

[35] G. Qi, "Modelings and mechanism analysis underlying both the $4 \mathrm{D}$ Euler equations and Hamiltonian conservative chaotic systems," Nonlinear Dynamics, vol. 95, no. 3, pp. 2063-2077, 2019.

[36] H. Bouadi, S. S. Cunha, A. Drouin, and F. Mora-Camino, "Adaptive sliding mode control for quadrotor attitude stabilization and altitude tracking," in Proceeding of the International Symposium on Computational Intelligence and Informatics, Budapest, Hungary, November 2011.

[37] Y. Yang and G. Qi, "Comparing mechanical analysis with generalized-competitive-mode analysis for the plasma chaotic system," Physics Letters A, vol. 383, no. 4, pp. 318-327, 2019.

[38] H. Goldstein, Classical Mechanics, Addison-Wesley, Boston, MA, USA, 1980.

[39] J. E. Marsden and T. Ratiu, Introduction to Mechanics and Symmetry: A Basic Ex-Position of Classical Mechanical Systems, Springer, Berlin, Germany, 2002.

[40] Q. Yang and G. Chen, "A chaotic system with one saddle and two stable node-foci," International Journal of Bifurcation and Chaos, vol. 18, no. 5, pp. 1393-1414, 2008. 


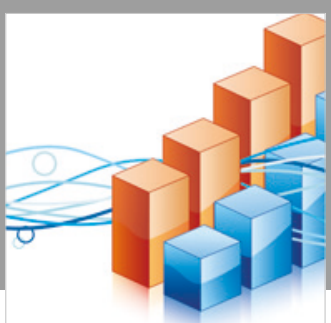

Advances in

Operations Research

\section{-n-m}
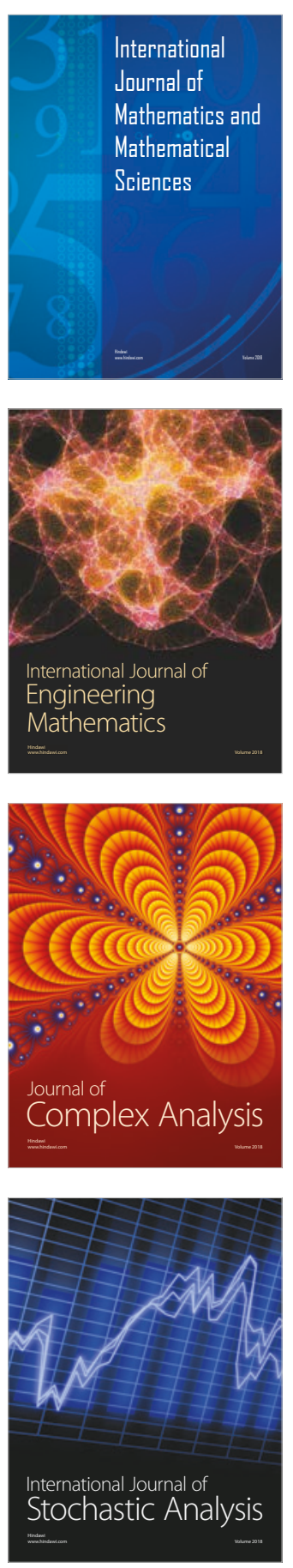
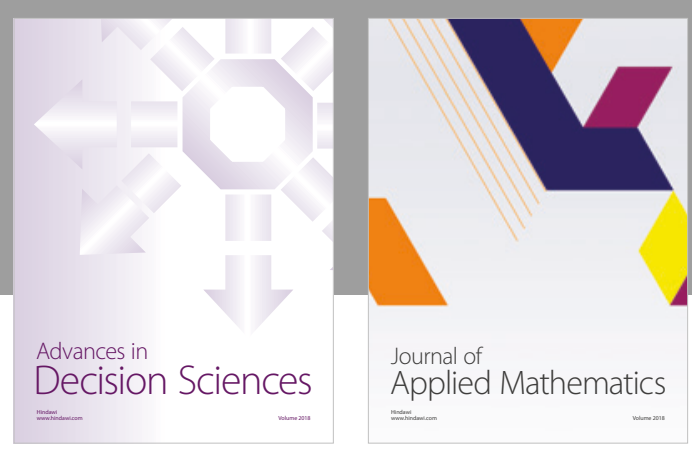

Journal of

Applied Mathematics
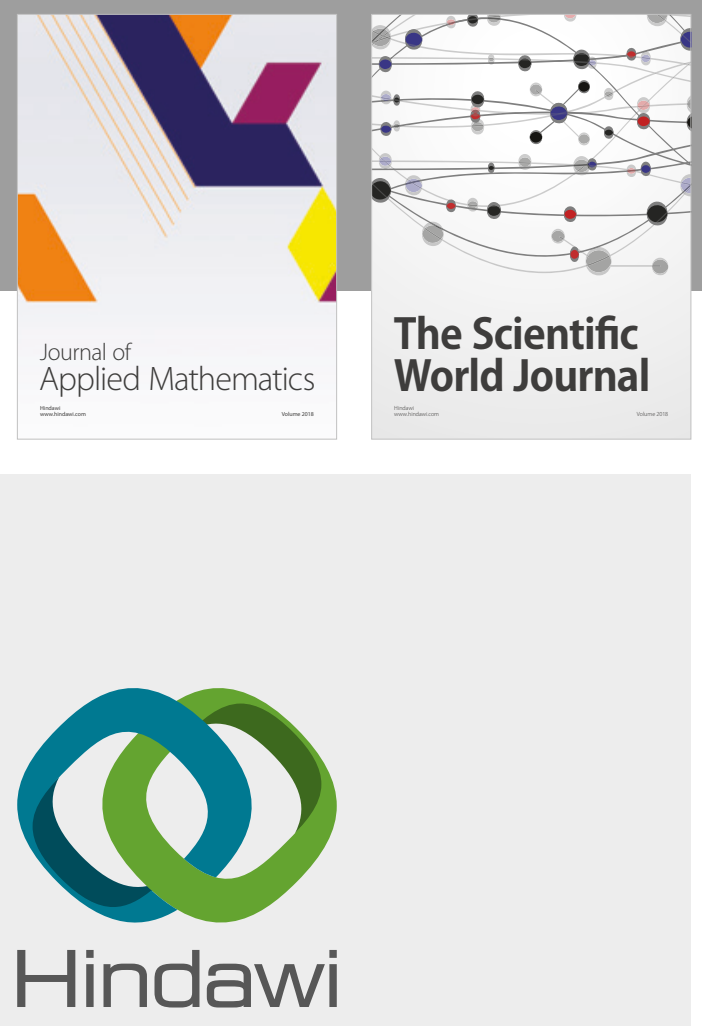

Submit your manuscripts at

www.hindawi.com

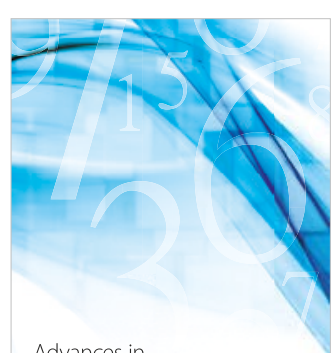

Advances in
Numerical Analysis
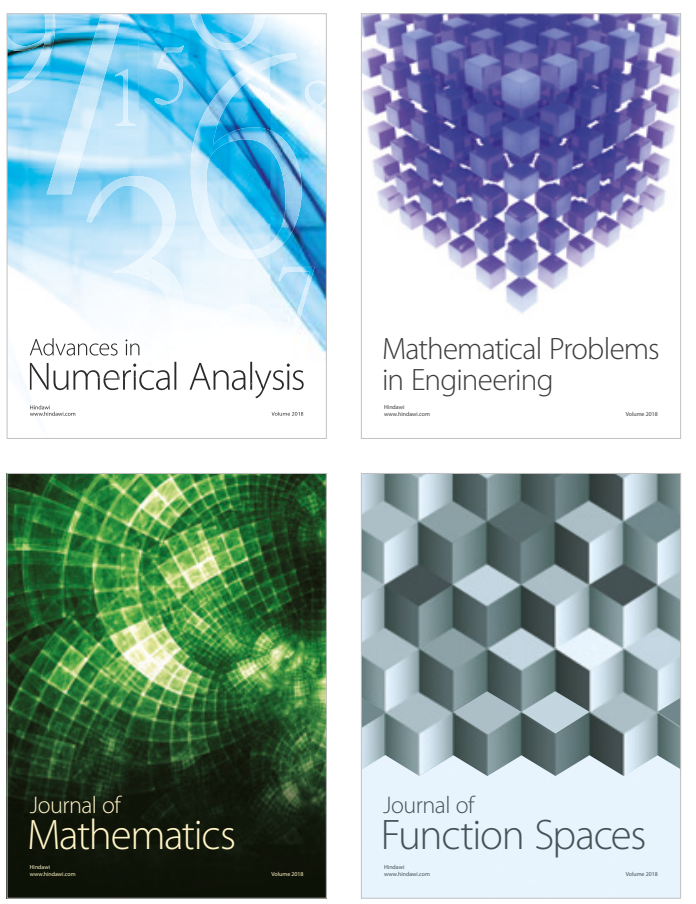

Mathematical Problems in Engineering

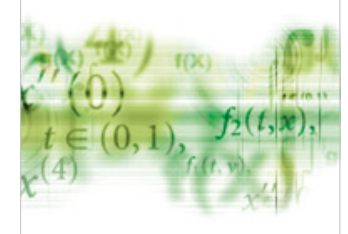

International Journal of

Differential Equations

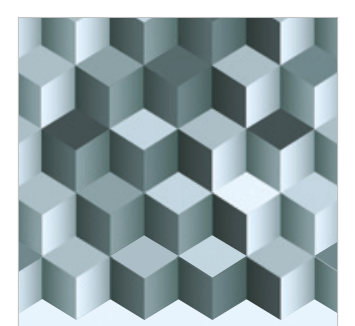

Journal of

Function Spaces
The Scientific

World Journal

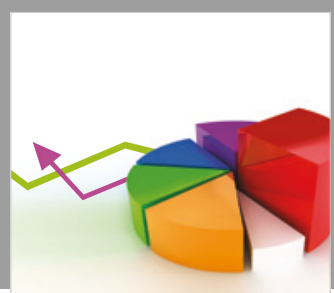

Journal of

Probability and Statistics
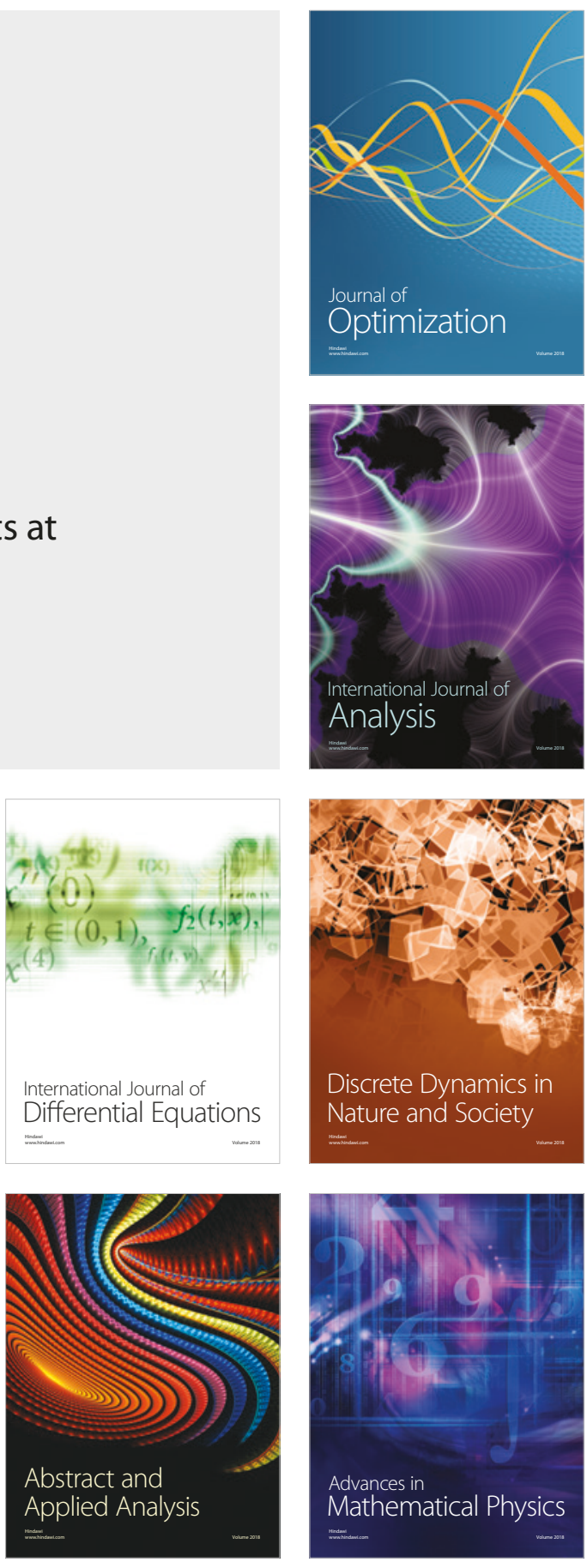